\title{
Discovery of a conserved rule behind the assembly of
}

\section{$\beta$-barrel membrane proteins.}

Edward M. Germany, ${ }^{1}$ Yue Ding, ${ }^{2,3}$ Kenichiro Imai, ${ }^{4}$ Rebecca S. Bamert, ${ }^{5,6}$ Rhys A. Dunstan, ${ }^{5,6}$ Yukari Nakajima, ${ }^{1}$ XiangFeng Lai, ${ }^{2}$ Chaille T. Webb, ${ }^{5,6}$ Christopher J. Stubenrauch, ${ }^{5,6}$ Kentaro Hidaka, ${ }^{1}$ Nakajohn Thewasano, ${ }^{1}$ Kher Shing Tan, ${ }^{5,6}$ Hsin-Hui Shen, ${ }^{2,3, *}$ Trevor Lithgow ${ }^{5,6, *}$ and Takuya Shiota ${ }^{1,7, *}$

${ }^{1}$ Organization for Promotion of Tenure Track, University of Miyazaki, Nishi 1-1 Gakuen Kibanadai, Miyazaki, 889-2192, Japan

${ }^{2}$ Department of Materials Science and Engineering, Monash University, Clayton, VIC 3800, Australia

${ }^{3}$ Biomedicine Discovery Institute and Department of Biochemistry and Molecular Biology, Monash University, Clayton 3800, Australia

${ }^{4}$ Cellular and Molecular Biotechnology Research Institute, National Institute of Advanced Industrial Science and Technology (AIST), 2-4-7 Aomi, Koto-ku, Tokyo 135-0064, Japan

${ }^{5}$ Centre to Impact AMR, Monash University, Clayton, VIC 3800, Australia

${ }^{6}$ Infection \& Immunity Program, Biomedicine Discovery Institute, and Department of Microbiology, Monash University, Clayton, VIC 3800, Australia

${ }^{7}$ Lead contact

*Correspondence: takuya_shiota@med.miyazaki-u.ac.jp (T.S.),

hsin-hui.shen@monash.edu (H.H.S.), trevor.lithgow@monash.edu (T.L.) 


\section{SUMMARY}

Gram-negative bacteria, mitochondria and chloroplasts contain outer membrane proteins (OMPs) characterized by a transmembrane domain with a $\beta$ barrel structure. Most OMPs have a diagnostic " $\beta$-signal" imprinted in the amino acid sequence of the final $\beta$-strand (-1 strand) of the $\beta$ barrel. Molecular chaperones of the Omp85 superfamily, such as BamA in the bacterial BAM complex, recognizes the $\beta$-signal and then catalyze the folding of the OMP into its membrane-embedded $\beta$ barrel structure. Here, we reconstituted OMP assembly in vitro to study this process with five model OMPs, revealing that a critical assembly signal exists in the fifth $\beta$-strand from the $\mathrm{C}$-terminus ( -5 strand). This signal was shown to drive a critical insertion step of the OMP assembly process but is not required for the initial recognition step between the OMP and BamA. Furthermore, we identified the receptor for this "-5 signal" as BamD, a second essential subunit of the BAM complex. Distinct binding sites for the $\beta$-signal and the -5 signal, were identified on BamD. Comparative sequence analysis showed that the -5 signal is a conserved feature in bacterial OMPs, and that mitochondrial OMPs also contains -5 signal motif positioned in the fifth from last strand of their $\beta$ barrel structure. We propose the "-5 rule" as a conserved feature of the process of OMP assembly in bacteria and the organelles of eukaryotes.

\section{HIGHLIGHTS}

- Gram-negative bacterial OMPs contain a conserved motif at the fifth $\beta$-strand from the Cterminus (-5 strand).

- The information encrypted in the conserved -5 signal is responsible for a rate-limiting step in protein insertion into the outer membrane

- Distinct binding sites on the outer membrane protein BamD recognize the -5 signal and a canonical $\beta$-signal.

- We propose -5 rule, to explain the function of the -5 signal in OMP assembly. 


\section{INTRODUCTION}

In Gram-negative bacteria and the organelles derived from them such as mitochondria and chloroplasts, outer membrane proteins (OMPs) play important roles in small molecule transport, protein import or export, and other membrane-mediated processes. The transmembrane domain of these OMPs are formed as a $\beta$-barrel: an intricate structure of amphiphilic $\beta$-strands in an anti-parallel array, where the first and last strand meet to form a cylinder. The fundamental process of OMP assembly is conserved across bacterial lineages and organelles, being mediated in each system by one or more chaperones of the Omp85 superfamily (Heinz and Lithgow, 2014; Pfanner et al., 2004; Ranava et al., 2018; Ulrich and Rapaport, 2015). Before its assembly into the outer membrane (OM), a nascent OMP is recognized by the assembly machinery by virtue of a $\beta$-signal, that has a conserved sequence motif ( $\Omega \times \Phi$ : aromatic residue / any residue / hydrophobic residue) in the final $\beta$-strand of the presumptive $\beta$-barrel (Kutik et al., 2008; Wang et al., 2021b). This $\beta$-signal interacts directly with the Omp85 superfamily chaperone during its assembly into the OM.

In bacteria, the essential protein translocase the Beta-barrel Assembly Machinery (BAM) complex contains the Omp85 superfamily protein, BamA (Wu et al., 2005). The subunit composition of the BAM complex varies in different bacterial lineages (Webb et al., 2012b), and in E. coli the BAM complex is composed of the one of the OMPs BamA, and four accessory lipoproteins: BamB, BamC, BamD and BamE (Bakelar et al., 2016; Gu et al., 2016; Iadanza et al., 2016). BamA and BamD are essential for viability in E coli and are found in all bacteria (Malinverni et al., 2006; Webb et al., 2012; Wu et al., 2005). BamA is integrated into the $\mathrm{OM}$ by a 16-stranded $\beta$-barrel and expose $\mathrm{N}$-terminal side into the periplasm with five polypeptide transport-associated (POTRA) domains (Noinaj et al., 2013; Voulhoux, 2003). BamD is docked to the POTRA domains of BamA, contributing to a large, funnel-like periplasmic feature of the BAM complex in the periplasm (Bakelar et al., 2016; Gu et al., 2016; Sandoval et al., 2011; Iadanza et al., 2016). BamD is composed of five TPR elements which enable its interactions with two other subunits, BamC and BamE, docking them to the BamA core (Kim et al., 2011, 2007; Malinverni et al., 2006). The BamB subunit has a distinct function, binding independently to the outer face of the funnel-like structure (Gu et al., 2016; Iadanza et al., 2016) to mediate the interactions that combine several BAM complexes into the active assembly precinct (Gunasinghe et al., 2018; Noinaj et al., 2017). While not essential for viability, the accessory subunits BamB, BamC and BamE are 
necessary to promote the maximum activity of the BAM complex in E. coli (Hagan et al., 2010; Gunasinghe et al., 2018).

The $\beta$-signal in a substrate polypeptide has been shown to interact with both BamD and BamA (Hagan et al., 2015; Tomasek et al., 2020; Xiao et al., 2021). While little is known about its interaction with BamD, the interaction of the $\beta$-signal with BamA has been shown to occur at the lateral gate formed between the $\mathrm{N}$-terminal strand and $\mathrm{C}$-terminal strand of BamA (Noinaj et al., 2013; Tomasek et al., 2020; Xiao et al., 2021). The lateral gate can adopt a closed-state with hydrogen bonds, or an unpaired opened-state. The recently reported structure of an assembly intermediate formed from a substrate captured in the BAM complex showed the $\beta$-signal of the substrate bound to N-terminal strand of BamA, forming a “asymmetric hybrid-barrel” in the process of budding (Tomasek et al., 2020).

Given the grave dangers associated with antimicrobial resistance (Tacconelli, 2017), several recent drug screens have identified lead peptidomimetic compounds capable of entering the lateral gate of BamA to exert a lethal effect on bacteria (Hart et al., 2019; Imai et al., 2019; Li et al., 2020; Luther et al., 2019; Sousa, 2019; Steenhuis et al., 2020). A recent structure of one of these compounds, darobactin, docked in the BAM complex mimiced the $\beta$-strand element of a nascent OMP, thereby blocking the site from accepting native substrates (Imai et al., 2019; Kaur et al., 2021; Xiao et al., 2021). Development of these projects, particularly to use in rational drug development, will need to further understand the mechanism of action.

Towards this goal, we employed in vitro reconstituted membrane assay using the E.coli Microsomal Membrane (EMM) (Gunasinghe et al., 2018). EMM assembly assay can chase throughout the assembly process by using ${ }^{35} \mathrm{~S}$-labelled substrate protein. After establishing conditions to measure the early stages of OMP capture and assembly, we used synthetic peptides to mimic the distinct regions of a model OMP, the major porin OmpC, to compete for substrate binding and assembly by the BAM complex. Two peptides have features that enable them to function as dominant substrates of the BAM complex. One of these is the C-terminal $\beta$-strand known to contain the $\beta$-signal for OMP recognition, and the other is a peptide representing the fifth from last $\beta$-strand ( -5 strand). The -5 strand peptide is highly competitive to the binding of OMPs and mutational analysis of OmpC confirmed that the information encrypted in this peptide is critical to BAM-mediated assembly of the OMP. Like the $\beta$-signal, part of the sequence in the -5 signal conforms to a $\Omega \times \Phi$ motif. Further analysis identified BamD as the critical receptor for the -5 signal, with site-specific photocrosslinking identifying distinct substrate recognition sites on BamD for the -5 signal and $\beta$ - 
signal. Mutational analysis showed that both of these substrate binding sites of BamD are important for OMP assembly. Protein-protein interaction studies and imaging of the active BAM complex in a membrane environment using neutron reflectometry (NR) revealed that mutations in the -5 signal cause OmpC to stall on the periplasmic domain of the BAM complex. Finally, comparative sequence analysis suggests that this motif in the -5 signal, is conserved in the $\beta$-barrel proteins of bacterial and mitochondria. We therefore propose a -5 rule for the process of $\beta$-barrel membrane protein recognition and assembly. 


\section{RESULTS}

\section{Peptidomimetics derived from E coli OmpC}

We employed EMM for in vitro reconstitution of BAM complex function, where the assembly of ${ }^{35} \mathrm{~S}$-labelled OmpC can be monitored over time (Gunasinghe et al., 2018). The EMM was isolated by sonication of $E$. coli followed by rapid high-speed centrifugation (Figure S1A). A synthetic peptide library was designed for coverage of the sequence features in OmpC, serving as a library of potential peptidomimetics (Figure S2A). The assembly of five OMPs were assessed in these assays: porins OmpC and OmpF, the OM-peptidoglycan bridge OmpA, the maltoporin LamB and the autotransporter (AT) EspP. ATs have two domains; (1) a $\beta$-barrel domain, assembled into the OM by the BAM complex, and (2) a passenger domain, which traverses the $\mathrm{OM}$ via the lumen of the $\beta$-barrel domain itself and is subsequently cleaved by correctly assembled $\beta$-barrel domain (Celik et al., 2012). Once the barrel domain is assembled into the membrane it becomes protease-resistant, with residual unassembled and passenger domains degraded (Leyton et al., 2014; Roman-Hernandez et al., 2014). Under the conditions of the EMM assay, EspP was observed to fragment as predicted (Figure 1A and S1B) and was used to rapidly screen inhibitory peptides in a robust highthroughput format.

Five peptides were found to inhibit EspP maturation (Figure S2B). Of these, peptide 23 corresponds to the final $\beta$-strand of $\mathrm{OmpC}$, which contains the information of the $\beta$-signal. Peptide 21 contains a similar $\Omega \times \Phi$ feature in the -3 strand (Hagan et al., 2015), as do three of the other four peptides (peptide 4, 18, and 21; Figure 1B). Alone, this motif is not sufficient to indicate inhibition, as peptide 10 does have sequence conforming to a $\Omega \times \Phi$ motif, but did not inhibit EspP assembly. Conversely peptide 17 lacked any recognizable $\Omega \times \Phi$ motif and inhibited EspP (Figure 1C).

To address whether the peptide-mediated inhibition was general, four other OMPs were assessed: OmpA, OmpC, OmpF and LamB (Burgess et al., 2008; Ureta et al., 2007). The assembly of OmpA can be monitored by heat modifiability, as folded OmpA is SDSresistant at room temperature thereby migrating faster on SDS-PAGE than the denatured form (Figure 1D). Monitoring the assembly of the three trimeric porins, OmpC, OmpF and LamB, required blue native $(\mathrm{BN})-\mathrm{PAGE}$ assessment of the trimeric form (Figure 1E-1G; "trimer"). As summarized in Figure $1 \mathrm{H}$, the assembly of OmpA into the membrane was slightly inhibited by peptides 4 and 17, whereas peptide 18 had comparable inhibition capacity to the $\beta$-signal peptide 23 (Figures $1 \mathrm{D}$ and $1 \mathrm{H}$ ). The assembly of OmpC was inhibited to the highest 
degree by peptide 18 and 23, with peptide 4 to less, and peptide 17 having slight inhibition (Figures $1 \mathrm{E}$ and $1 \mathrm{H}$ ). The assembly of OmpF followed a similar pattern of inhibition (Figure $1 \mathrm{~F}$ and $1 \mathrm{H}$ ). LamB showed a similar trend of inhibition by peptides, with peptide 18 and 23 displaying greatest inhibition and peptide 4 and 17 a slight inhibition (Figures $1 \mathrm{G}$ and $1 \mathrm{H}$ ). Taken together, the peptide screens suggest that the assembly of the various OMPs is responsive to the presence of peptide mimetics, and that there are sequence features in the peptides for differential inhibitory action on the BAM complex in a bacterial membrane environment.

\section{Mutations in the $\beta$-strands of OmpC impact on assembly.}

Sequence analysis indicated a conservation of specific residues, to determine the sequencespecificity of the inhibition by the peptides, we systematically mutated these conserved residues in OmpC (Figure 2A). Point mutations in the peptide 4 region did not impair assembly of $\mathrm{OmpC}$, whereas $\mathrm{OmpC}$ carrying mutations in conserved residues within regions defined by peptide 17, 18, 21, or 23 dramatically reduced the assembly of OmpC (Figure 2A). In the $\Omega \times \Phi$ motif in the $\beta$-signal peptide 23, Y365 corresponds to $\Omega$ and the Y365A mutant OmpC showed greatly reduced assembly (Figure 2B). Peptide 21 corresponds to the $-3 \beta$ strand, while the region encompassed by peptides 17 and 18 over-lap in the $-5 \beta$-strand of OmpC. F280A and Y286A mutants corresponding to conserved residues in these peptides, showed severe loss of assembly (Figure $2 \mathrm{~B}$ ). The $\beta$-signal, the -3 strand, and the -5 strand have the same orientation, traversing the OM from cell surface toward the periplasm with each strand containing a hydrophilic amino acid in position "x" of the $\Omega x \Phi$ motif. In all cases, the $\Omega \times \Phi$ motif is positioned at the periplasm-outer membrane interface (Figure 2C).

It was intriguing to discover an important assembly determinant in the $-5 \beta$-strand, as it is substantially distant from the C-terminal region that engages with the BAM complex to initiate what is considered to be a passive strand-by-strand assembly reaction (Tomasek and Kahne, 2021). Thus, we analyzed the -5 strand in more detail, focusing on conserved residues present in the $-5 \beta$-strand. Sequence conservation analysis suggested conservation in the equivalent $\beta$-strands of OMPs whose structures have been determined (Figure S3 and S4). Based on this general information, we constructed mutants in these conserved residues in OmpF and LamB (Figure 2C). Mutation of conserved residues in the -5 strand greatly reduced the assembly of OmpF and LamB (Figure 2D and 2E). Analysis via BN-PAGE showed the formation of a high-molecular-weight product during assay of the mutant forms of these OMPs (Figure 2B, 2D, 2E). 
We sought to determine whether F280 and Y286 of OmpC were necessary for assembly in vivo. Steady state protein levels and the oligomeric state was determined for OmpC, and for the variants harboring single or double alanine mutations at the Y286 and F280 sites. Expression of FLAG-OmpC variants induced by $0.1 \%$ arabinose was confirmed through immunoblotting of total cell lysate (Figure 3A and 3B). In the respective EMM fractions, the protein levels of FLAG-OmpC variants were equivalent, but with a marked reduction seen in the steady-state level of the double ("FY") mutant OmpC(F280A, Y286A) (Figure 3C). On BN-PAGE analysis, the FLAG-OmpC runs as two forms (Figure 3D), with the upper form corresponding in size to the native trimer (Figure 3E). Dimeric forms of OmpC have been seen previously, and shown to have structural and functional properties equivalent to trimers (Rocque and McGroarty, 1989). The FY mutant displayed drastic reduction of the dimer and trimer (Figure 3D). The expression of FY mutant FLAG-OmpC influenced the steady-state level of the endogenous trimeric OmpF (Figure 3E), suggesting that it impacts activity of the BAM complex. As a control to this, the level and integrity of the BAM complex was monitored and found to be equivalent in all conditions (Figures 3C and $3 \mathrm{~F})$. Taken together these results suggest that OMPs contain assembly information encrypted in the -5 strand. We termed this the "-5 signal".

\section{BamD recognizes the -5 signal.}

To determine the receptor for the -5 signal using His-tagged components of the BAM complex. In vitro binding assays showed that both BamA and BamD can independently bind to the OmpC polypeptide (Figure S5). To characterize this binding activity, we assessed the impact of peptides 17 and 18, each of which contains the -5 signal, in these binding assays (Figure 4A). Both peptide 17 and peptide 18 inhibited binding of $\mathrm{OmpC}$ to $\mathrm{BamD}$, but not to BamA.

Continuing this investigation, in vitro site-specific photo-crosslinking was applied to determine the substrate-binding region of BamD (Figure 4B). A library of $40 \mathrm{BamD}$ variants was created wherein at different positions the non-natural amino acid, $p$-benzoyl- $\mathrm{L}^{-}$ phenylalanine (BPA), was introduced by suppressor tRNA method in E. coli cells (Chin et al., 2002). The 40 variants of BamD were purified, individually incubated with ${ }^{35}$ S-labeled OmpC, followed by irradiation with ultraviolet (UV) light to induce the formation of a crosslink in near-neighbour residues (Figures 4B and 4C). The UV-dependent appearance of crosslinks to OmpC occurred at 17 different positions in BamD (Figure S9A and 4D). The analysis was repeated to compare the extent of BamD cross-links formed with three different 
${ }^{35}$ S-labelled OmpC variants: WT, the -5 signal mutant Y286A, or the $\beta$-signal mutant Y365A, Q366A, F367A (Figure S9C and 5E). The mutation in the -5 signal decreased the crosslinked product between OmpC(Y286A) and the N-terminal region of BamD (residues 62 and 65), while mutations in the $\beta$-signal in OmpC(Y365A, Q366A, F367A) decreased the crosslinked product in the C-terminal positions of BamD (residues 195, 200 and 204) (Figure 4E and 4F).

Using these sites as landmarks, mapping of sequence conservation across the surface of BamD revealed conserved residues in both the $\mathrm{N}$ - and C-terminus, overlapping with residues identified via crosslinking (Figure 5A). Using this sequence conservation, we constructed two mutant forms of BamD. In the first, BamD(Y62A), a conserved tyrosine residue in the proposed -5 signal binding site was replaced with alanine. In the second mutant, $\mathrm{BamD}(\mathrm{R} 197 \mathrm{~A})$, a conserved arginine residue in the proposed $\beta$-signal binding site was replaced. We sought to establish the importance of the -5 signal binding site, using the $\beta$ signal binding site as a baseline.

A bamD depletion strain with endogenous bamD expression controlled by an arabinose-responsive promoter was transformed with a plasmid encoding either of these mutant proteins (Figure 5B and S11). Growth conditions were established wherein endogenous BamD was depleted (Figure S7A-S7E). Neither the BamD(Y62A) nor the BamD(R197A) mutants affected growth or steady-state protein levels of other components of the BAM complex or the major porins (Figure 5C, S8A), nor assembly of the BAM complex (Figure 5D, S8B).

After determination of time point that deplete endogenous BamD and still not disturbing cell growth (Figure S8A), membrane fractions were prepared from the four strains of E. coli, transformed to express: wild-type (WT) BamD, BamD(Y62A), BamD(R197A) or no BamD (vector). The EMM assembly assay showed that the -5 binding site region was as important as the $\beta$-signal binding site to the overall assembly rates observed for OmpF (Figure 5E), OmpC (Figure 5F), and LamB (Figure 5G). These results suggest that the BamD is the receptor for the -5 signal and BamD recognizes -5 signal and $\beta$-signal at the $\mathrm{N}$-terminal and C-terminal side, respectively.

\section{Reconstitution of OmpC interaction with the BAM complex.}

The finding that a region of BamD interacts selectively with an internal $\beta$-strand ( -5 strand) of OmpC (Figure 4E) added to three observations suggesting that mutations in the -5 strand of OmpC cause a delayed interaction with the BAM complex: (i) that a peptidomimetic of the -5 strand reduces the BAM-mediated assembly of multiple $\beta$-barrel proteins (Figure $1 \mathrm{H}$ ), (ii) 
that a high molecular weight ("Int") form is observed on BN-PAGE during assembly of these $\beta$-barrel proteins where the -5 strand is mutated (Figure $2 \mathrm{~B}, \mathrm{D}, \mathrm{E}$ ), and (iii) that expression of the FY form of OmpC with the -5 strand mutated impacts on the steady-state level of the endogenous porin. We sought to define and characterize binding of the -5 signal to BamD in the context of the membrane-embedded BAM complex.

A "gel shift" BN-PAGE analysis was used to directly determine if the high molecular weight form of ${ }^{35} \mathrm{~S}$-labelled OmpC-Y286A was in contact with the BAM complex. The addition of an antibody recognizing the surface-exposed component BamC to detergent solubilized EMM fraction prior to electrophoresis, resulted in a dramatic shift (i.e. retardation due to the added mass of antibodies) of the intermediate ("Int"), consistent with OmpCY286A being a stable assembly intermediate engaged with the BAM complex (Figure 6A). To determine when this assembly intermediate formed, we performed urea extraction after EMM assembly assay and analyzed the urea-resistant pellet by BN-PAGE. The trimer form of OmpC was not extracted by urea, indicating that correctly assembled $\mathrm{OmpC}$ is fully inserted into OM (Figure 6B). The intermediate was completely extracted with urea, as expected for a substrate protein engaged in protein-protein interactions with the EMM fraction (i.e. the BAM complex). These results suggest that the mutant OmpC interacts with the BAM complex, but is not yet inserted into the membrane environment of the EMM.

Neutron reflectometry (NR) is a powerful tool for probing interactions and dynamics of substrate-binding to membrane-embedded proteins (Ding et al., 2020; Shen et al., 2014). BamA containing a hexa-histidine tag at the surface exposed loop 4 (Chen et al., 2021; Ding et al., 2020) was immobilized on an Ni-NTA atomic flat gold-coated silicon wafer to orient the POTRA domain distal to the silicon wafer, and a lipid layer was reconstituted to integrate BamA into a membrane-like environment (Figure 6C). To evaluate these additions (Figure 6D), we sequentially measured: (i) BamA with lipid (1st measurement), (ii) BamA with lipid and BamD (2nd measurement), and (iii) BamA-BamD-lipid with the addition of OmpC(Y286A) (3rd measurement). Comparing the reflectivity profiles of three measurements (Figure 6D), the addition of BamD and OmpC showed a shift of fringe to low $\mathrm{Q}$ range around 0.02-0.03 $\AA^{-1}$, indicating changes in the layers after $\mathrm{BamD}$ and OmpC(Y286A) additions.

In previous analyses of the whole BAM complex and of sub-complexes (Chen et al., 2021; Ding et al., 2020), the periplasmic domains of BamA were treated as two rigid bodies: POTRA1-2(P1-2) and POTRA3-5(P3-5). Thus, we divided BamA into four sub-layers for data fitting: a His6 extra-cellular layer, a membrane layer (containing the $\beta$-barrel domain of 
BamA), a POTRA3-5 layer and a POTRA1-2 layer. Analysis of the data showed that the thickness of layers at each measurement (Figure 6E, S9A, S9B). At the 2nd measurement, the SLD profiles showed that BamD was located in the P3-5 layer (Figure 6E, S9C, S9D), an observation consistent with previous studies on the resting BAM complex (Chen et al., 2021; Ding et al., 2020). The 3rd measurement, on addition of the OmpC(Y286A) substrate, revealed it bound to only the P3-5 layer, and that this stimulated the extension of this layer from $29.7 \pm 0.8 \AA$ to $45.5 \pm 1.6 \AA$ (Figure $6 \mathrm{E} \mathrm{S9E}, \mathrm{S} 9 \mathrm{~F}$ ). These findings indicate that OmpC(Y286A) is stacked at what would be the periplasmic region of the BAM consistent with the -5 signal normally assisting the membrane insertion step of OMP assembly. 


\section{DISCUSSION}

\section{The -5 signal for assembly of OMPs}

Early work suggested that a C-terminal bulky hydrophobic residue of a bacterial OMP, usually a phenylalanine or tryptophan, was important for $\beta$-barrel assembly into the bacterial outer membrane (OM) (Bitto and McKay, 2003; de Cock et al., 1997; Jansen et al., 2000; Robert et al., 2006) In an unbiased motif analysis of 1523 diverse AT protein sequences the $\beta$ signal motif was defined as a contextual motif conforming to $G / A-x-x-G-x-R / G-\Omega-x-\Phi$ (where " $x$ " represents a non-conserved residue) (Celik et al., 2012), with the final residue (Ф) in the $\beta$-signal motif was always a bulky hydrophobic residue, usually a phenylalanine or tryptophan. Recent studies have showen that $\beta$-signal interacts with the lateral gate of BamA (Doyle and Bernstein, 2019; Hagan et al., 2015; Tomasek et al., 2020; Xiao et al., 2021), suggesting that the $\beta$-signal is the primary segment to be inserted via the lateral gate. In the present study we find that, in addition to the $\beta$-signal, bacterial OMPs also contain a $\beta$-signallike sequence repeated in the -5 strand, which is related to a rate-limiting step of OMPs insertion of assembly.

In consideration of the process of bacterial OMP assembly, and for consistency with the work discussed below, we suggest that the definition of the $\beta$-signal be refined to it being the C-terminal segment of the nascent, OMP polypeptide with conserved sequence features that enable its recognition by the C-terminal domain of BamD, and that it is the first element of the nascent OMP polypeptide to then engage in the lateral gate of BamA. By definition then, the -5 signal is in the fifth last $\beta$-strand of the nascent OMP polypeptide, with conserved sequence features that enable its recognition by the $\mathrm{N}$-terminal domain of $\mathrm{BamD}$, in order to assist translocation of the nascent OMP polypeptide into the periplasmic funnel of the BAM complex.

Revision of the literature found that three previous studies had unknowingly addressed the -5 strand of OMPs, providing valuable information on the assembly pathway for OmpA and EspP [(Wang et al., 2021b; Ieva et al., 2011)]. Recently, mutations to the -5 strand of OmpA resulted in a loss of assembly ability, suggesting the importance of the context for the -5 signal in the -5 strand (Wang et al., 2021b). Motif analysis of ATs found that while the $\beta$-signal motif was found in the final strand, a similar motif was found in the 8th strand, the -5 strand, of the 12-stranded ATs (Celik et al., 2012). Likewise, in studying the assembly of EspP, introduction of BPA at position F1214, the aromatic residue in the -5 signal, cross-linked with BamD during the course of EspP assembly (Ieva et al., 2011; Wang et al., 
2021b). Taken together with our finding that the assembly of OmpA and EspP are both prone to inhibition by peptides mimicking the -5 strand of OmpC, suggests that information encrypted in the -5 strand of these three proteins is important for insertion into the OM via the BAM complex.

\section{The -5 rule of OMPs}

Mitochondria evolved from bacteria, and the mitochondrial protein import pathway is driven by a set of molecular machines that are of modular construction, several derived from the bacterial ancestor (Chacinska et al., 2009; Dolezal et al., 2006). In mitochondria, the Sorting and Assembly Machinery (SAM complex) is responsible for the assembly of OMPs into the OM (Diederichs et al., 2020; Takeda et al., 2021; Wang et al., 2021a). The SAM complex is homologous to, and evolved from, the BAM complex inherited from bacterial endosymbiont ancestor of mitochondria (Gentle et al., 2004; Pfanner et al., 2004). Mitochondrial OMPs engage the SAM complex by at a $C$-terminal $\beta$ signal. The mitochondrial $\beta$-signal conforms to the motif $\zeta-\mathrm{x}-\mathrm{G}-\mathrm{x}-\mathrm{x}-\Phi-\mathrm{X}-\Phi$ (where $\zeta$ is a polar residue and $\Phi$ is a hydrophobic residue), and is necessary for OMPs assembly by the SAM complex (Kutik et al., 2008). Previous studies on bacterial OMPs had set the stage for the characterization of the mitochondrial and bacterial $\beta$-signals. While there is general conservation between the BAM and SAM complexes and the $\beta$ signal targeting mechanism, we found that the mitochondrial $\beta$ signal was incapable of inhibiting the BAM complex (Figure S2). We evaluated mitochondrial OMP sequences to find that the mitochondrial $\beta$-barrel proteins, Tom40 and Mdm10, also contain what appears a mitochondrial $\beta$-signal-like sequence repeated in the -5 strand (Figure S10). Therefore, we propose a -5 rule, in which a $\beta$-signal-like motif is present in the -5 strand of OMPs, in both mitochondria and bacteria.

\section{BamD is the receptor for the $\beta$-signal and the $-\mathbf{5}$ signal}

BamD is responsible for two important functions; (i) it provides the major surface for the BamCDE module of the BAM complex to engage with BamA (Bergal et al., 2016; Gu et al., 2016; Iadanza et al., 2016; Kim et al., 2011, 2007; Malinverni et al., 2006), and (ii) it directly binds substrate proteins (Ieva et al., 2011; Lee et al., 2018; Wang et al., 2021b). Our current study showes that BamD recognizes two distinct features of substrate proteins, both the -5signal and the $\beta$-signal. The -5 signal binds in the $\mathrm{N}$-terminal region of $\mathrm{BamD}$, while the $\beta$ signal binds via residues in the $\mathrm{C}$-terminal region of BamD. In the BAM complex structure, 
the N-terminal region of BamD interacts with the BamA POTRA1 and POTRA2 (P1-2 hinge), while the C-terminal region interacts with BamA POTRA5 in proximity to the lateral gate (Bakelar et al., 2016; Gu et al., 2016). NR analysis revealed that OmpC(Y286A), which has a non-functional -5 signal, fails to bind POTRA1-2 of BamA and instead accumulates at the POTRA3-5 layer. The accumulated substrate also had the impact of extending this region of the BamA outwards from the membrane by $15 \AA$ (from $30 \AA$ to $45 \AA$ ). As the signal-binding residues of BamD are located on the interior side of the funnel-like structure formed by BamD and POTRA domains (Figure 7A, 7B), substrate protein should bind to the interior cavity of this structure. The interior of the funnel formed by the P5-BamD-P1-2 hinge is capable of accommodating a substantial amount of a substrate polypeptide (Figure 7C, 7D). Thus, while the funnel region may allow for rapid translocation of small regions of polypeptide that bind either in the -5 signal site or the $\beta$-signal site, it might alternatively provide a means for the C-terminal 5-6 strands of a nascent OMP polypeptide to marshal on $\mathrm{BamD}$, in the BAM funnel prior to initiation of $\beta$-signal transfer into the lateral gate. In summary, multiple binding sites for peptides representing amphipathic $\beta$-strands exist in the luminal surface of the funnel structure leading into the lateral gate of the BAM complex.

\section{The role of -5 signal for OMP assembly}

In this study, we show recognition of the -5 signal by BamD is important for membrane insertion of substrates from the POTRA3-5 soluble domain of the BAM complex, closest to the membrane. Two signal binding regions of BamD sit slightly wider than fully folded final five strands of substrate (Figure 7C, 7D). Thus, BamD may bundle the final 5 strands, initiating the formation of hydrogen bonds between $\beta$-hairpins and the preliminary $\beta$-barrel. Partially folded OMPs then fix the direction of the lipid-facing hydrophobic side and hydrophilic barrel lumen, accelerating insertion into the membrane. A previous in vitro study showed that BamD itself helped BamA assembly into liposomes, also supports this hypothesis (Hagan et al., 2010).

The current debate centres on two distinct models to describe the mechanism of OMP assembly: (i) In the BamA-assisted mechanism (Plummer and Fleming, 2016; Ranava et al., 2018; Wu et al., 2020), the OMP polypeptide is completely or largely folded before it engages with the BAM complex and utilizes the lateral gate movements to destabilize the lipids to promote an environment into which the OMP can embed into the plane of the membrane. (ii) In the Budding mechanism (Ranava et al., 2018; Tomasek and Kahne, 2021; Wu et al., 2020), the completely or largely unfolded OMP polypeptide engages with the BAM complex, and 
the primary function of the lateral gate movements are to stitch together the $\beta$-strands of the substrate, with the growing OMP budding away from BamA in the membrane plane.

Ranava et al., 2018 have proposed a systems-approach to understanding OMP biogenesis, allowing that some OMPs might be more prone to "budding" into the membrane, while others might have features that simply require assistance into the membrane. Whether the underlying mechanisms are general or more substrate-specific, two recently-acquired lines of evidence need to be satisfied: (i) that the lateral gate can engage stably with the $\beta$ signal (Xiao et al., 2021), and (ii) that at least five of the C-terminal $\beta$-strands of a nascent OMP polypeptide might queue in the BAM funnel when the -5 strand is interacting with BamD. The $\beta$-signal recognition site of BamD is close to POTRA5, proximal to the lateral gate (Figure 7). This geometric arrangement would enable direct entry of the partially folded substrate $\beta$-signal, from BamD, into lateral gate.

The findings on the role of the -5 signal in this study shows that binding of a conserved, internal segment of the nascent polypeptide to the BamD subunit of the BAM complex is a crucial step of OMP folding and assembly. Moreover, the EMM assembly assay, used for peptidomimetic, is a powerful tool not only for analysis of the function of $\beta$-barrel membrane protein assembly, but also screening and characterization of BAM complex inhibitors.

\section{Limitations of the Study}

Although the EMM assembly assay is a powerful tool to study OMP assembly, as a reduced system, it possibly lacks soluble protein factors which assist in the assembly process. Additionally, NR can provide atomic resolution of protein localization, but cannot determine the secondary structure, thus it is unclear the folding state of the substrate protein at the periplasmic domain of the BAM complex. Since BamD-substrate interaction is transient, we employed purified BamD for cross-linking study. This artificial condition might catch nonspecific binding such as substrate binding to interface for subunit binding of BamD. Although from mutational analysis of BamD we believe at least two regions of BamD are important for substrate binding. 


\section{SUPPLEMENTAL INFORMATION}

Supplemental Information includes Extended Experimental Procedures, 11 figures, and 10 tables.

\section{AUTHOR CONTRIBUTION}

E.M.G., T.L., and T.S. designed research and wrote the paper. E.M.G. and T.S. performed most of the key experiments. Y.D, E.G. and T.S. measured NR and Y.D., X.F.L and H.-H.S. analyzed NR data. Y.N., K.H., and K.S.T. prepared materials. R.A.D., C.T.W., and C.J.S supervised NR sample preparation. N.T. performed BamD-depletion experiments. K.I performed sequence analysis. R.S.B prepared materials and edited the paper.

\section{ACKNOWLEDGEMENTS}

We thank the members of the Shiota, Shen and Lithgow labs for discussion and critical comments on the manuscript. This work was supported by JSPS KAKENHI to T.S. (19K16077, 18KK0197 and 18H06052), to E.M.G. (21K15043), and to K.I. (JP18K11543 and 21H03551), JST FOREST Program to T.S. (JPMJFR2064), AMED Platform Project of Basis for Supporting Innovative Drug Discovery and Life Science Research (BINDS) to K.I. (JP21am0101114), Australian Research Council (DP160100227) to T.L, and National Health and Medical Research Council (CDF1106798) to H.-H.S.. The following grants are also acknowledged; a grant from the Ichiro Kanehara Foundation for the Promotion of Medical Sciences and Medical Care, Waksman Foundation of Japan, Tokyo Biochemical Research Foundation, Sumitomo Foundation, Naito Foundation, Uehara Memorial Foundation, The Shinnihon Foundation of Advanced Medical Treatment Research, and Noguchi institute (to T.S.). The NR experiment at the J-PARC MLF was performed under a user program (Proposal No. 2019B0364). We thank N. L. Yamada for support of NR measurements, and H. Nishitoh for access to instruments (for T.S.). Radiation experiment were performed at RI unit of FSRC of UoM. 


\section{REFERENCES}

Bakelar, J., Buchanan, S.K., and Noinaj, N. (2016). The structure of the $\beta$-barrel assembly machinery complex. Science $351,180-186$.

Bergal, H.T., Hopkins, A.H., Metzner, S.I., and Sousa, M.C. (2016). The Structure of a BamA-BamD Fusion Illuminates the Architecture of the $\beta$-Barrel Assembly Machine Core. Structure 24, 243-251.

Bitto, E., and McKay, D.B. (2003). The periplasmic molecular chaperone protein SurA binds a peptide motif that is characteristic of integral outer membrane proteins. J Biol Chem 278, 49316-49322.

Burgess, N.K., Dao, T.P., Stanley, A.M., and Fleming, K.G. (2008). $\beta$-Barrel Proteins That Reside in the Escherichia coli Outer Membrane in Vivo Demonstrate Varied Folding Behavior in Vitro. Journal of Biological Chemistry 283, 26748-26758.

Celik, N., Webb, C.T., Leyton, D.L., Holt, K.E., Heinz, E., Gorrell, R., Kwok, T., Naderer, T., Strugnell, R.A., Speed, T.P., et al. (2012). A bioinformatic strategy for the detection, classification and analysis of bacterial autotransporters. PLoS One 7, e43245.

Chacinska, A., Koehler, C.M., Milenkovic, D., Lithgow, T., and Pfanner, N. (2009). Importing Mitochondrial Proteins: Machineries and Mechanisms. Cell 138, 628-644.

Chen, X., Ding, Y., Bamert, R.S., Le Brun, A.P., Duff, A.P., Wu, C.-M., Hsu, H.-Y., Shiota, T., Lithgow, T., and Shen, H.-H. (2021). Substrate-dependent arrangements of the subunits of the BAM complex determined by neutron reflectometry. Biochimica et Biophysica Acta (BBA) - Biomembranes 1863, 183587.

Chin, J.W., Martin, A.B., King, D.S., Wang, L., and Schultz, P.G. (2002). Addition of a photocrosslinking amino acid to the genetic code of Escherichia coli. Proceedings of the National Academy of Sciences 99, 11020-11024.

de Cock, H., Struyvé, M., Kleerebezem, M., van der Krift, T., and Tommassen, J. (1997). Role of the carboxy-terminal phenylalanine in the biogenesis of outer membrane protein PhoE of Escherichia coli K-12. J Mol Biol 269, 473-478.

Diederichs, K.A., Ni, X., Rollauer, S.E., Botos, I., Tan, X., King, M.S., Kunji, E.R.S., Jiang, J., and Buchanan, S.K. (2020). Structural insight into mitochondrial $\beta$-barrel outer membrane protein biogenesis. Nat Commun 11, 3290.

Ding, Y., Shiota, T., Le Brun, A.P., Dunstan, R.A., Wang, B., Hsu, H.-Y., Lithgow, T., and Shen, H.-H. (2020). Characterization of BamA reconstituted into a solid-supported lipid bilayer as a platform for measuring dynamics during substrate protein assembly into the membrane. Biochimica et Biophysica Acta (BBA) - Biomembranes 1862, 183317.

Dolezal, P., Likic, V., Tachezy, J., and Lithgow, T. (2006). Evolution of the molecular machines for protein import into mitochondria. Science 313, 314-318. 
Doyle, M.T., and Bernstein, H.D. (2019). Bacterial outer membrane proteins assemble via asymmetric interactions with the BamA $\beta$-barrel. Nat Commun 10, 3358.

Gentle, I., Gabriel, K., Beech, P., Waller, R., and Lithgow, T. (2004). The Omp85 family of proteins is essential for outer membrane biogenesis in mitochondria and bacteria. J Cell Biol $164,19-24$.

Gu, Y., Li, H., Dong, H., Zeng, Y., Zhang, Z., Paterson, N.G., Stansfeld, P.J., Wang, Z., Zhang, Y., Wang, W., et al. (2016). Structural basis of outer membrane protein insertion by the BAM complex. Nature 531, 64-69.

Gunasinghe, S.D., Shiota, T., Stubenrauch, C.J., Schulze, K.E., Webb, C.T., Fulcher, A.J., Dunstan, R.A., Hay, I.D., Naderer, T., Whelan, D.R., et al. (2018). The WD40 Protein BamB Mediates Coupling of BAM Complexes into Assembly Precincts in the Bacterial Outer Membrane. Cell Reports 23, 2782-2794.

Hagan, C.L., Kim, S., and Kahne, D. (2010). Reconstitution of Outer Membrane Protein Assembly from Purified Components. Science 328, 890-892.

Hagan, C.L., Wzorek, J.S., and Kahne, D. (2015). Inhibition of the $\beta$-barrel assembly machine by a peptide that binds BamD. Proceedings of the National Academy of Sciences 112, 2011-2016.

Hart, E.M., Mitchell, A.M., Konovalova, A., Grabowicz, M., Sheng, J., Han, X., RodriguezRivera, F.P., Schwaid, A.G., Malinverni, J.C., Balibar, C.J., et al. (2019). A small-molecule inhibitor of BamA impervious to efflux and the outer membrane permeability barrier. Proc Natl Acad Sci U S A 116, 21748-21757.

Iadanza, M.G., Higgins, A.J., Schiffrin, B., Calabrese, A.N., Brockwell, D.J., Ashcroft, A.E., Radford, S.E., and Ranson, N.A. (2016). Lateral opening in the intact $\beta$-barrel assembly machinery captured by cryo-EM. Nat Commun 7, 12865.

Ieva, R., Tian, P., Peterson, J.H., and Bernstein, H.D. (2011). Sequential and spatially restricted interactions of assembly factors with an autotransporter domain. Proceedings of the National Academy of Sciences 108, E383-E391.

Imai, Y., Meyer, K.J., Iinishi, A., Favre-Godal, Q., Green, R., Manuse, S., Caboni, M., Mori, M., Niles, S., Ghiglieri, M., et al. (2019). A new antibiotic selectively kills Gram-negative pathogens. Nature 576, 459-464.

Jansen, C., Heutink, M., Tommassen, J., and de Cock, H. (2000). The assembly pathway of outer membrane protein PhoE of Escherichia coli. Eur J Biochem 267, 3792-3800.

Kaur, H., Jakob, R.P., Marzinek, J.K., Green, R., Imai, Y., Bolla, J.R., Agustoni, E., Robinson, C.V., Bond, P.J., Lewis, K., et al. (2021). The antibiotic darobactin mimics a $\beta$ strand to inhibit outer membrane insertase. Nature 593, 125-129. 
Kim, K.H., Aulakh, S., and Paetzel, M. (2011). Crystal Structure of $\beta$-Barrel Assembly Machinery BamCD Protein Complex. Journal of Biological Chemistry 286, 39116-39121.

Kim, S., Malinverni, J.C., Sliz, P., Silhavy, T.J., Harrison, S.C., and Kahne, D. (2007). Structure and Function of an Essential Component of the Outer Membrane Protein Assembly Machine. Science 317, 961-964.

Kutik, S., Stojanovski, D., Becker, L., Becker, T., Meinecke, M., Krüger, V., Prinz, C., Meisinger, C., Guiard, B., Wagner, R., et al. (2008). Dissecting Membrane Insertion of Mitochondrial $\beta$-Barrel Proteins. Cell 132, 1011-1024.

Lee, J., Sutterlin, H.A., Wzorek, J.S., Mandler, M.D., Hagan, C.L., Grabowicz, M., Tomasek, D., May, M.D., Hart, E.M., Silhavy, T.J., et al. (2018). Substrate binding to BamD triggers a conformational change in BamA to control membrane insertion. Proc Natl Acad Sci USA 115, 2359-2364.

Leyton, D.L., Johnson, M.D., Thapa, R., Huysmans, G.H.M., Dunstan, R.A., Celik, N., Shen, H.-H., Loo, D., Belousoff, M.J., Purcell, A.W., et al. (2014). A mortise-tenon joint in the transmembrane domain modulates autotransporter assembly into bacterial outer membranes. Nature Communications 5.

Li, Y., Zhu, X., Zhang, J., Lin, Y., You, X., Chen, M., Wang, Y., Zhu, N., and Si, S. (2020). Identification of a Compound That Inhibits the Growth of Gram-Negative Bacteria by Blocking BamA-BamD Interaction. Front Microbiol 11, 1252.

Luther, A., Urfer, M., Zahn, M., Müller, M., Wang, S.-Y., Mondal, M., Vitale, A., Hartmann, J.-B., Sharpe, T., Monte, F.L., et al. (2019). Chimeric peptidomimetic antibiotics against Gram-negative bacteria. Nature 576, 452-458.

Malinverni, J.C., Werner, J., Kim, S., Sklar, J.G., Kahne, D., Misra, R., and Silhavy, T.J. (2006). YfiO stabilizes the YaeT complex and is essential for outer membrane protein assembly in Escherichia coli: YfiO assembles OMPs and stabilizes the YaeT complex. Molecular Microbiology 61, 151-164.

Noinaj, N., Kuszak, A.J., Gumbart, J.C., Lukacik, P., Chang, H., Easley, N.C., Lithgow, T., and Buchanan, S.K. (2013). Structural insight into the biogenesis of $\beta$-barrel membrane proteins. Nature 501, 385-390.

Noinaj, N., Gumbart, J.C., and Buchanan, S.K. (2017). The $\beta$-barrel assembly machinery in motion. Nat Rev Microbiol 15, 197-204.

Pfanner, N., Wiedemann, N., Meisinger, C., and Lithgow, T. (2004). Assembling the mitochondrial outer membrane. Nat Struct Mol Biol 11, 1044-1048.

Plummer, A.M., and Fleming, K.G. (2016). From Chaperones to the Membrane with a BAM! Trends in Biochemical Sciences 41, 872-882. 
Ranava, D., Caumont-Sarcos, A., Albenne, C., and Ieva, R. (2018). Bacterial machineries for the assembly of membrane-embedded $\beta$-barrel proteins. FEMS Microbiol Lett 365.

Robert, V., Volokhina, E.B., Senf, F., Bos, M.P., Van Gelder, P., and Tommassen, J. (2006). Assembly factor Omp85 recognizes its outer membrane protein substrates by a speciesspecific C-terminal motif. PLoS Biol 4, e377.

Rocque, W.J., and McGroarty, E.J. (1989). Isolation and preliminary characterization of wild-type OmpC porin dimers from Escherichia coli K-12. Biochemistry 28, 3738-3743.

Roman-Hernandez, G., Peterson, J.H., and Bernstein, H.D. (2014). Reconstitution of bacterial autotransporter assembly using purified components. ELife 3.

Sandoval, C.M., Baker, S.L., Jansen, K., Metzner, S.I., and Sousa, M.C. (2011). Crystal Structure of BamD: An Essential Component of the $\beta$-Barrel Assembly Machinery of GramNegative Bacteria. Journal of Molecular Biology 409, 348-357.

Selkrig, J., Mosbahi, K., Webb, C.T., Belousoff, M.J., Perry, A.J., Wells, T.J., Morris, F., Leyton, D.L., Totsika, M., Phan, M.-D., et al. (2012). Discovery of an archetypal protein transport system in bacterial outer membranes. Nat Struct Mol Biol 19, 506-510, S1.

Shen, H.-H., Leyton, D.L., Shiota, T., Belousoff, M.J., Noinaj, N., Lu, J., Holt, S.A., Tan, K., Selkrig, J., Webb, C.T., et al. (2014). Reconstitution of a nanomachine driving the assembly of proteins into bacterial outer membranes. Nature Communications 5.

Sousa, M.C. (2019). New antibiotics target the outer membrane of bacteria. Nature 576, 389390 .

Steenhuis, M., Ten Hagen-Jongman, C.M., van Ulsen, P., and Luirink, J. (2020). StressBased High-Throughput Screening Assays to Identify Inhibitors of Cell Envelope Biogenesis. Antibiotics (Basel) 9, E808.

Tacconelli, E. (2017). GLOBAL PRIORITY LIST OF ANTIBIOTIC-RESISTANT BACTERIA TO GUIDE RESEARCH, DISCOVERY, AND DEVELOPMENT OF NEW ANTIBIOTICS. 7.

Takeda, H., Tsutsumi, A., Nishizawa, T., Lindau, C., Busto, J.V., Wenz, L.-S., Ellenrieder, L., Imai, K., Straub, S.P., Mossmann, W., et al. (2021). Mitochondrial sorting and assembly machinery operates by $\beta$-barrel switching. Nature 590, 163-169.

Tomasek, D., and Kahne, D. (2021). The assembly of $\beta$-barrel outer membrane proteins. Current Opinion in Microbiology 60, 16-23.

Tomasek, D., Rawson, S., Lee, J., Wzorek, J.S., Harrison, S.C., Li, Z., and Kahne, D. (2020). Structure of a nascent membrane protein as it folds on the BAM complex. Nature 583, 473478. 
Ureta, A.R., Endres, R.G., Wingreen, N.S., and Silhavy, T.J. (2007). Kinetic Analysis of the Assembly of the Outer Membrane Protein LamB in Escherichia coli Mutants Each Lacking a Secretion or Targeting Factor in a Different Cellular Compartment. Journal of Bacteriology $189,446-454$.

Urfer, M., Bogdanovic, J., Lo Monte, F., Moehle, K., Zerbe, K., Omasits, U., Ahrens, C.H., Pessi, G., Eberl, L., and Robinson, J.A. (2016). A Peptidomimetic Antibiotic Targets Outer Membrane Proteins and Disrupts Selectively the Outer Membrane inEscherichia coli. Journal of Biological Chemistry 291, 1921-1932.

Voulhoux, R. (2003). Role of a Highly Conserved Bacterial Protein in Outer Membrane Protein Assembly. Science 299, 262-265.

Wang, Q., Guan, Z., Qi, L., Zhuang, J., Wang, C., Hong, S., Yan, L., Wu, Y., Cao, X., Cao, J., et al. (2021a). Structural insight into the SAM-mediated assembly of the mitochondrial TOM core complex. Science eabh0704.

Wang, X., Peterson, J.H., and Bernstein, H.D. (2021b). Bacterial Outer Membrane Proteins Are Targeted to the Bam Complex by Two Parallel Mechanisms. MBio 12.

Webb, C.T., Heinz, E., and Lithgow, T. (2012a). Evolution of the $\beta$-barrel assembly machinery. Trends Microbiol 20, 612-620.

Webb, C.T., Selkrig, J., Perry, A.J., Noinaj, N., Buchanan, S.K., and Lithgow, T. (2012b). Dynamic Association of BAM Complex Modules Includes Surface Exposure of the Lipoprotein BamC. Journal of Molecular Biology 422, 545-555.

Wu, R., Stephenson, R., Gichaba, A., and Noinaj, N. (2020). The big BAM theory: An open and closed case? Biochim Biophys Acta Biomembr 1862, 183062.

Wu, T., Malinverni, J., Ruiz, N., Kim, S., Silhavy, T.J., and Kahne, D. (2005). Identification of a Multicomponent Complex Required for Outer Membrane Biogenesis in Escherichia coli. Cell 121, 235-245.

Xiao, L., Han, L., Li, B., Zhang, M., Zhou, H., Luo, Q., Zhang, X., and Huang, Y. (2021). Structures of the $\beta$-barrel assembly machine recognizing outer membrane protein substrates. FASEB j. 35. 


\section{FIGURE LEGENDS}

Figure 1. Peptides derived from the OMP, OmpC, are capable of inhibiting BAM complex assembly

(A) ${ }^{35} \mathrm{~S}$-labelled EspP was synthesized by rabbit reticulocyte lysate, then incubated with EMM. At the indicated time, the EMM samples were shifted onto ice to halt assembly reaction, and treated with (+) or without (-) Proteinase K (see Extended Fig. 1). Proteins were analyzed by SDS-PAGE and radio-imaging. (p) and (m) indicate to precursor and mature form of EspP, respectively.

(B) Sequence of the active peptides, with residues in the $\Omega \times \Phi$ motif colored magenta.

(C) Peptides with inhibition (Figure S2) were incubated with ${ }^{35}$ S-labelled EspP in the presence of EMM. Densitometry data from 3 independent EspP assembly assays were plotted versus time to quantify inhibition.

(D) ${ }^{35} \mathrm{~S}$-labelled OmpA was incubated with EMM then shifted onto ice to halt the assembly reaction at indicated times. Samples were split and incubated at either $25^{\circ} \mathrm{C}$ or $99^{\circ} \mathrm{C}$ for 10 min, before analysis by SDS-PAGE and radio-imaging. (u) and (f) indicate unfolded or folded, respectively.

(E)-(G) Peptide inhibition of assembly reactions for OmpC (E), OmpF (F) and LamB (G). ${ }^{35}$ S-labelled proteins were incubated as above for indicated time, shifted onto ice, and solubilized in BN-PAGE lysis buffer containing 1.5\% DDM. Solutions were clarified via centrifugation and analyzed by BN-PAGE and radio-imaging. The assembled, trimeric form of each porin is indicated.

(H) Densitometry data for the endpoints of the assays on all assembled proteins (D)-(G), from 3 independent experiments.

\section{Figure 2. The $-5 \beta$-strand of OMPs is Necessary for Proper OMP Assembly}

(A) (Top) Representation of OmpC, annotated to show the position of each $\beta$-strand (blue arrows) and the region mimicked in the indicated peptides. (Middle) Sequence logos show the conservation of sequence for the residues in these regions (see Methods), where the height of letter corresponds to the degree of conservation across bacterial species, residues are color-coded: aromatic (orange), hydrophobic (green), basic (blue), acidic (red), polar (sky blue) and proline and glycine (black). (Bottom) EMM assembly assay of OmpC mutants: ${ }^{35} \mathrm{~S}-$ labelled OmpC wild-type or mutant proteins were incubated with EMM for 30 or 90 min, and analyzed by BN-PAGE and radio-imaging. 
(B) ${ }^{35}$ S-labelled OmpC or OmpC mutant proteins: the $-5 \beta$-strand (F280 and Y286) or final $\beta$ strand (Y325 and Y365), were incubated with EMM for 10, 30 or $90 \mathrm{~min}$, and then analyzed by BN-PAGE and radio-imaging. Int. indicates intermediate.

(C) Structure-informed sequence characteristics of the $-5 \beta$-strand of OmpC, OmpF, and LamB. Residues highlighted in gray represent hydrophobic resides, with black boxes indicating residues in the $\Omega \times \Phi$ motif. Color code of amino acid as in (A).

(D), (E) Assembly assay of ${ }^{35}$ S-labelled OmpF (D) and ${ }^{35}$ S-labelled LamB (E) with mutations to corresponding residues in the $-5 \beta$-strand as in $\mathrm{B}$.

\section{Figure 3. Mutations to OmpC -5 $\beta$-strand Results in Assembly Defects in vivo}

(A) Schematic model of FLAG-OmpC expression system in E. coli. The plasmid-borne copy of $O m p C$ was mutated and the mutant proteins are selectively detected with antibodies to the FLAG epitope.

(B) Total cell lysates were prepared from the variant E. coli strains expressing FLAG-OmpC (WT) or the -5 strand mutants: F280A, Y286A, double mutant F280A/Y286A (FY) with (+) or without (-) arabinose. The steady-state levels of the indicated proteins were assessed after SDS-PAGE by immunoblotting with anti-FLAG antibody.

(C) After growth with arabinose induction, EMM fraction was isolated from each of the FLAG-OmpC variant expressing E. coli strains to assess steady state levels of OmpC variants and factors mediating OMP biogenesis: BamA, BamB, BamC, BamD, and BamE, and the periplasmic chaperone SurA, by SDS-PAGE and immunoblotting.

(D) - (F) EMM isolated as in C, were solubilized with $1.5 \%$ DDM and subjected to BNPAGE and immunoblotting. The indicated proteins were detected by anti-FLAG (D), anti$\mathrm{OmpF} / \mathrm{C}(\mathrm{E})$ and anti-BamA antibodies, respectively.

\section{Figure 4. The $\mathrm{N}$ - and $\mathrm{C}$-terminus of BamD Interacts with Substrate}

(A) Ni-NTA was used to affinity purify His6-BamA or His6-BamD. The Ni-NTA beads were then incubated in the presence $(+)$ or absence $(-)$ of inhibitor peptide 17 or 18 , with ${ }^{35} \mathrm{~S}$ labelled OmpC. T- 5\% total input, F- unbound fraction, W- wash fraction, E- Eluted fraction. The graph represents densitometry analysis of data from 3 independent experiments.

(B) Schematic of in vitro ${ }^{35} \mathrm{~S}$-labelled OmpC-BamD crosslinking. BamD containing BPA was purified, incubated with the ${ }^{35} \mathrm{~S}$-labelled OmpC, and irradiated with UV light to induce crosslinking. 
(C) Autoradiograph of BamD-OmpC crosslinking. UV irradiation (+) induces cross-link products migrating at $\sim 90 \mathrm{kDa}$ as indicated by the pink arrows, and asterisks indicate nonspecific species that were not dependent on UV irradiation. Amino acid positions in BamD listed above.

(D) Structure of BamD, highlighting the amino acid positions of BPA incorporation that cross-linked with OmpC (orange spheres) and BPA positions that did not form cross-linking products (violet spheres).

(E) Comparative crosslink of three variants of OmpC: OmpC (WT), OmpC(Y286A) (-5), and OmpC(Y365A, Q366A, F367A) ( $\beta$ ).

(F) Structure of BamD, highlighting the amino acid positions of BPA incorporation that cross-linked with OmpC which failed in the -5 signal mutant OmpC(Y286A) or with the $\beta$ signal mutant OmpC(Y365A, Q366A, F367A).

\section{Figure 5. Residues in the $\mathrm{N}$ - and $\mathrm{C}$-terminal of BamD are crucial for proper BAM complex function}

(A) Sequence conservation analysis of BamD. Target residues, Y62 and R197, are indicated with dark pink spheres on the crystal structure of BamD, sequence logos represent conservation in the immediate region of these residues.

(B) Schematic depiction of the BamD depletion strain of E. coli used to express mutant BamD proteins.

(C) Steady state levels of BamD mutant strains in EMM. Protein levels of BAM complex proteins, and OmpF were stable between WT, Y62A, R197A mutants. Wedge indicates that in each case, two samples were assessed corresponding to either $4 \mu \mathrm{g}$ or $12 \mu \mathrm{g}$ total EMM protein.

(D) Pull-down assay of the variants of BamD-His8. The EMMs as in (C) were solubilized with $1.5 \%$ DDM and then subjected to Ni-NTA. T- 5\% total input, F- unbound fraction, Wwash fraction, E- Eluted fraction. Each fraction was analyzed by SDS-PAGE and immunoblotting against indicated antibodies.

(E) - (G) ${ }^{35}$ S-labelled OmpF (E), ${ }^{35}$ S-labelled OmpC (F) and ${ }^{35}$ S-labelled LamB (G) were incubated with EMM as in (C), and then analyzed by BN-PAGE and radio-imaging.

Figure 6. Mutations to the $-5 \beta$-strand results in formation of a high molecular weight intermediate species 
(A) BN-PAGE analysis of ${ }^{35}$ S-labelled OmpC(Y286A) incubated with EMM for 60 mins. The EMM fraction was solubilized with 1.5\% DDM and incubated with (+) or without (-) anti-BamC antibody for $40 \mathrm{~min}$ and the samples analyzed by BN-PAGE. The presumptive translocation intermediate (Int) and the gel-shift species (Int $+\mathrm{AB})$ are indicated.

(B) ${ }^{35}$ S-labelled OmpC was incubated with EMM and then analyzed by BN-PAGE and radioimaging. EMM were then washed and solubilized in the presence $(+)$ or absence $(-)$ of $6 \mathrm{M}$ urea, after which the membrane fraction was re-isolated via ultra-speed centrifugation for 45 min. Isolated membranes were solubilized in 1.5\% DDM and analyzed by BN-PAGE.

(C) Flow-chart of NR experiment. Chromium (Cr) and gold coated silicon-wafers were initially treated with Ni-NTA followed by the addition of purified His6-BamA. Lipid membrane, POPC, was add for the $1^{\text {st }}$ measurement, after which BamD was incubated for 30 minutes for the $2^{\text {nd }}$ measurement. Finally, unfolded OmpCY286A was added to the wafer for the $3^{\text {rd }}$ measurement.

(D) NR profiles for BamA in membrane ( $1^{\text {st }}$ measurement, black line), BamAD (2 ${ }^{\text {nd }}$ measurement, red line), and BamAD-substrate ( $3^{\text {rd }}$ measurement, blue line).

(E) Table summarizes of the thickness, volume fraction, and roughness of NR analysis. $1^{\text {st }}$, $2^{\text {nd }}$, and $3^{\text {rd }}$ measurement displayed in black, magenta, and blue, respectively. P3-5: POTRA3, POTRA 4, and POTRA5, P1-2: POTRA1 and POTRA2, Lipid: POPC, Solution: D2O, gold match water (GMW) and H2O. Details were described in Table S8 to S10.

(F) Thickness of P3-5 layer from the three measurements defined by NR analysis in (E). Thickness of P3-5 was unaffected with the addition of BamD. Upon the addition of OmpC(Y286A), P3-5 layer increased thickness from 30 to $60 \AA$.

\section{Figure 7. Model of recognition of -5 -signal and $\beta$-signal by the periplasmic domain of the BAM complex}
(A) Structure of substrate engaged BAM complex (PDBID: 6V05).
(B) Bottom-up view of the periplasmic domain of complex in (A).
(C) Cavity formed by POTRA 1-2 and 5 of BamA and BamD can accommodate C-terminus

five strands of OmpC. final strand and -5 strand are indicated light blue and orange, respectively. The signal binding region of BamD is emphasized by deep purple. PDBID; BAM complex, 5DOQ and OmpC 2J1N.

(D) Bottom-up view of complex in (C). 
bioRxiv preprint doi: https://doi.org/10.1101/2021.10.29.466387; this version posted October 29, 2021. The copyright holder for this preprint (which was not certified by peer review) is the author/funder. All rights reserved. No reuse allowed without permission.

A

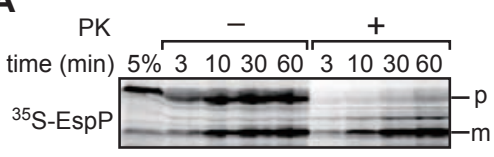

B Peptide 4 68-TDQLTGYGQWEYQIQGNS-85 Peptide 17 263-YNATRVGSLGWANKAQNF-280 Peptide 18 278-QNFEAVAQYQFDFGLRPS-295 Peptide 21 308-ATYYFNKNMSTYVDYKIN-325 Peptide 23 353-INTDNIVALGLVYQF-367

C

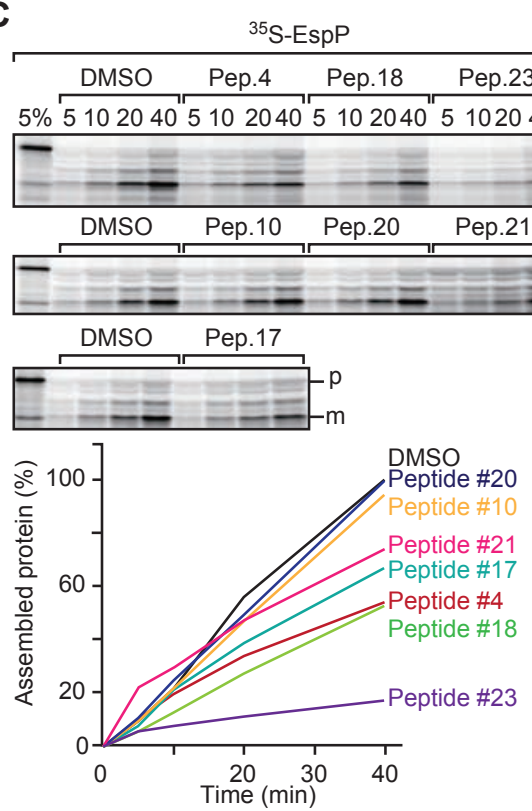

D

\begin{tabular}{|c|c|c|c|c|c|c|c|c|c|c|}
\hline \multirow[b]{3}{*}{ Boil } & \multicolumn{8}{|c|}{${ }^{35} \mathrm{~S}-\mathrm{OmpA}$} & \multirow{2}{*}{\multicolumn{2}{|c|}{ Pep.4 }} \\
\hline & DM & ISO & Pep & .17 & & .18 & Pep. & & & \\
\hline & + & - & + & - & + & - & + & - & + & - \\
\hline $5 \%$ & 310 & 1310 & 1310 & 1310 & 1310 & & 13310 & 1310 & 1310 & 131 \\
\hline
\end{tabular}

E

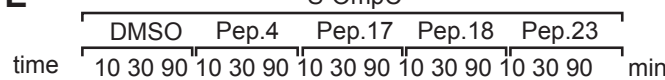

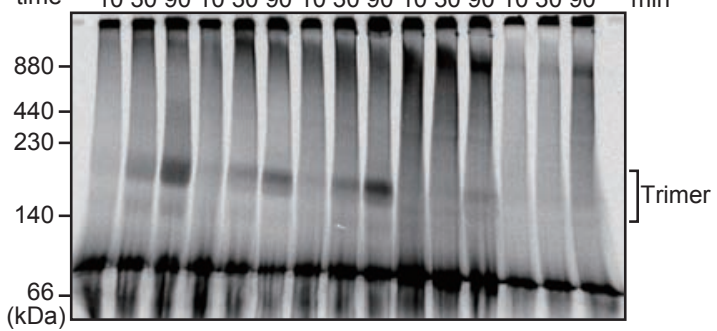

F

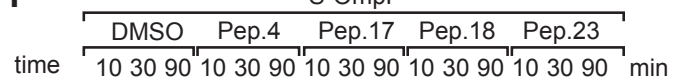

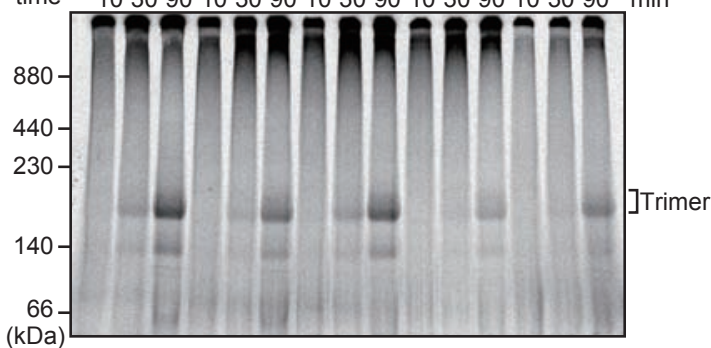

$(\mathrm{kDa})$

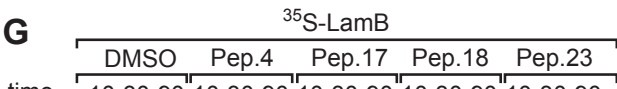
time $103090103090103090 " 103090103090$ min

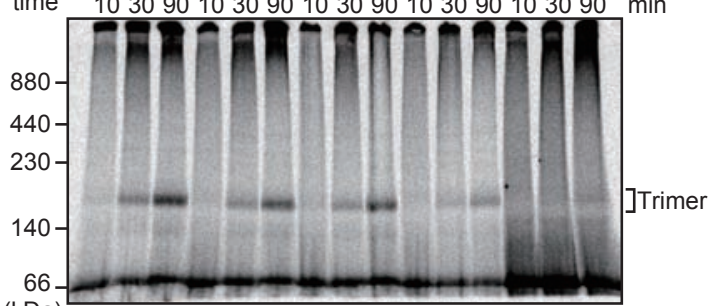


A A OmpC mature part (22 - 367 aa)

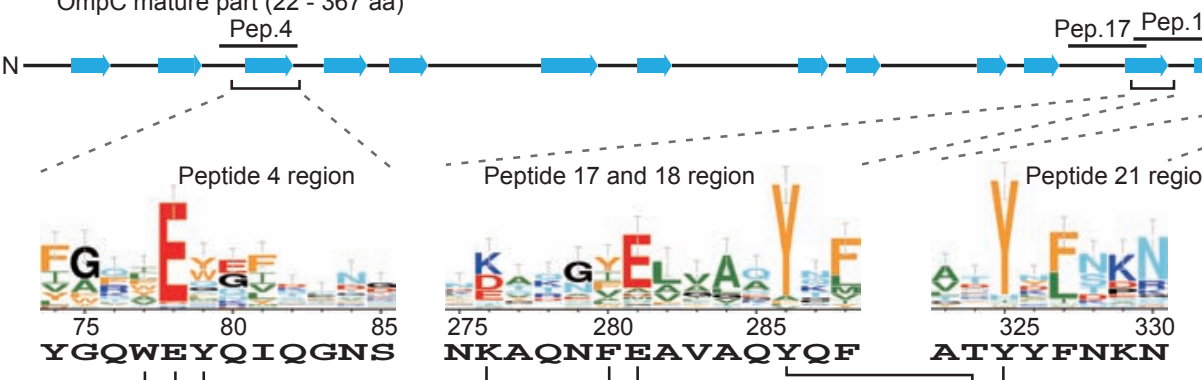

† ।

† ATYYFNKN

WT W77A E78A E78K Y79A K276A K276E F280A F280V E281A E281K Y286A P294A Y325A Y365A WT

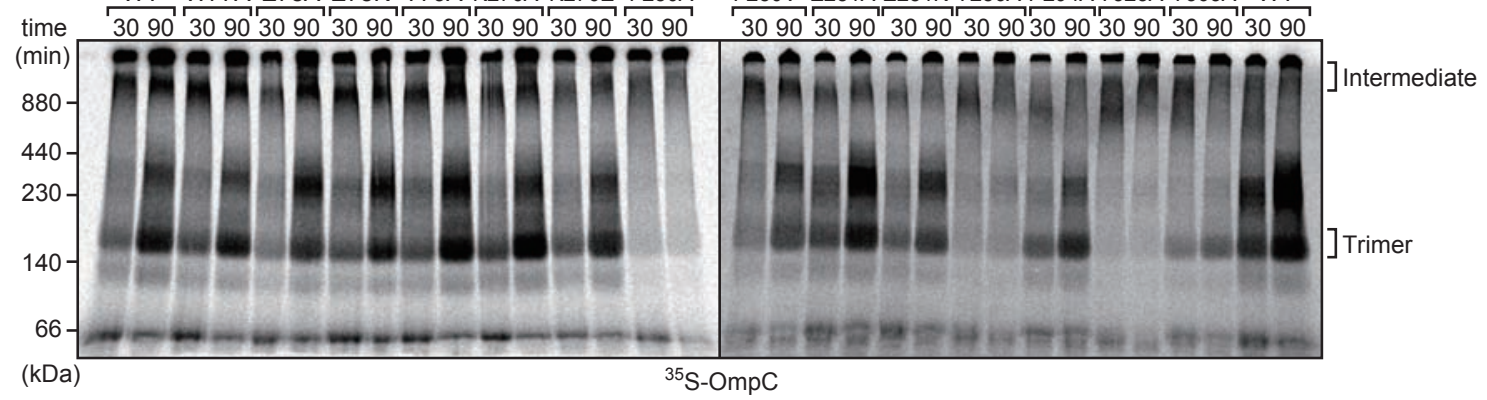

B

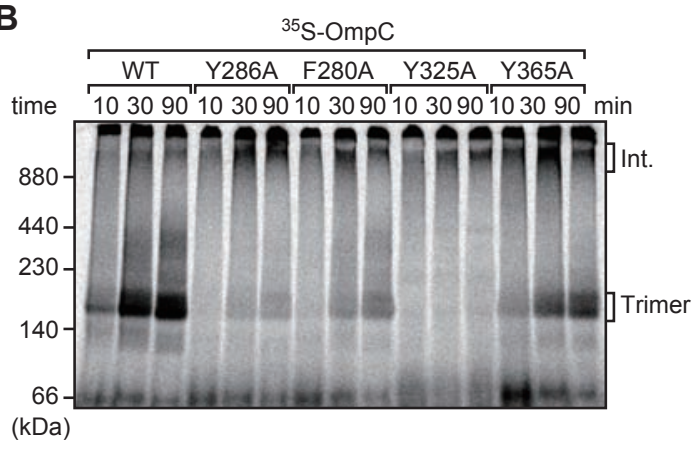

C

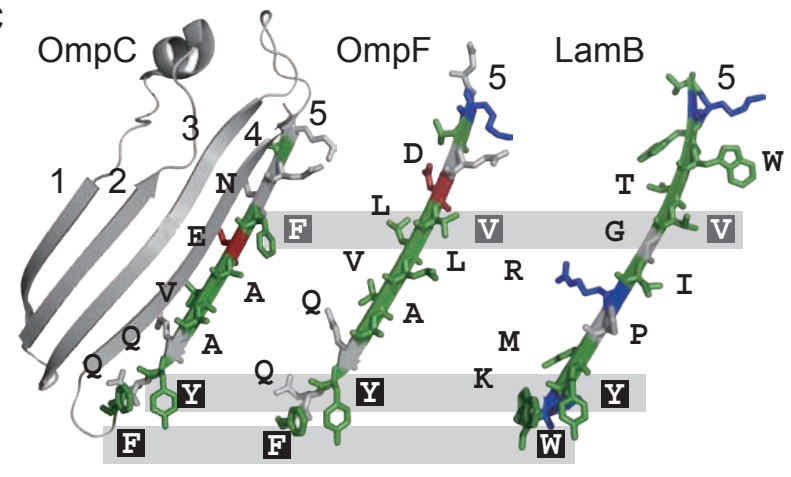

E

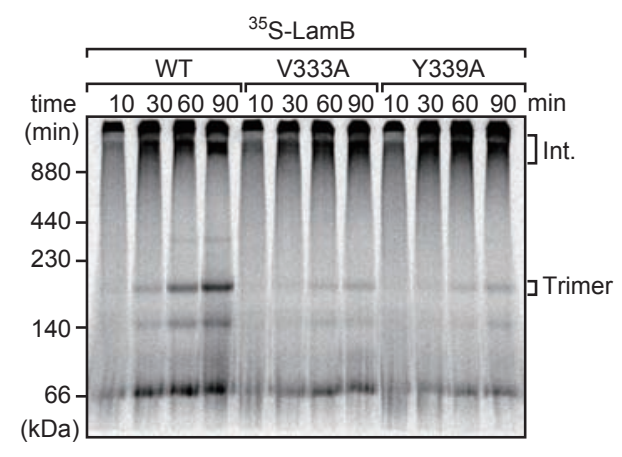


A

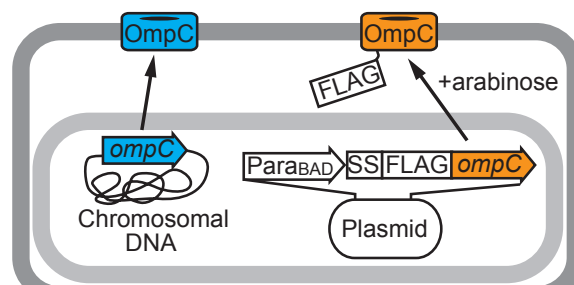

B

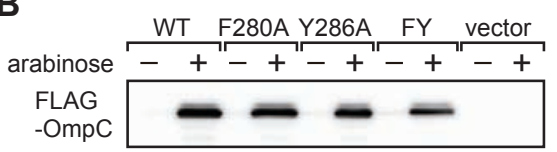

C

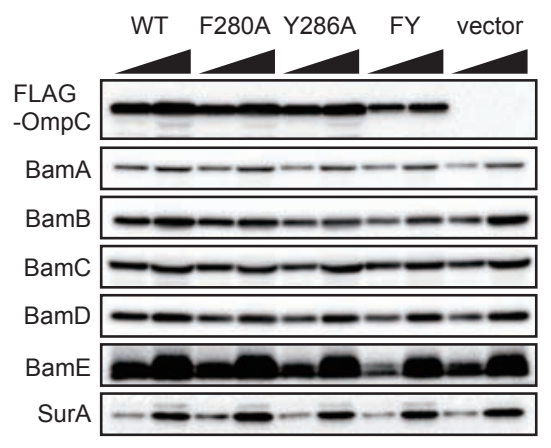

$\mathbf{F}$

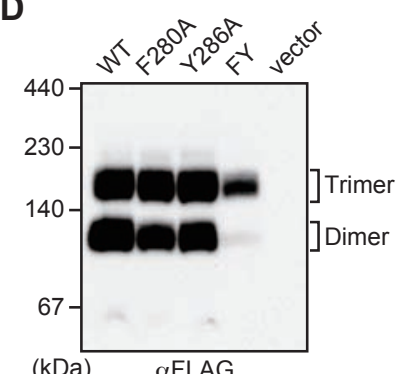

$\mathbf{E}$

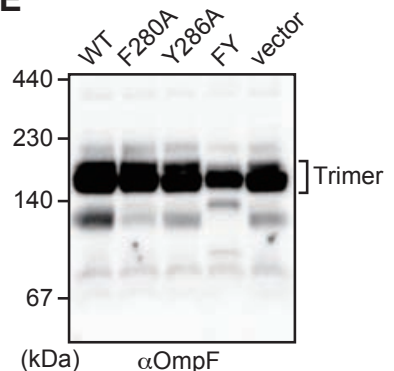

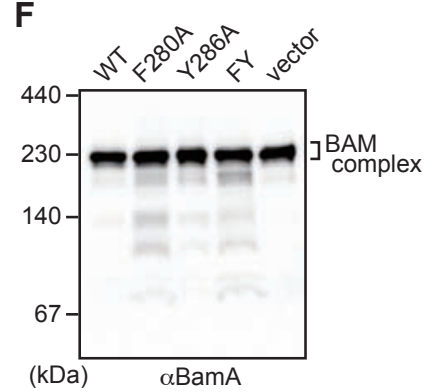




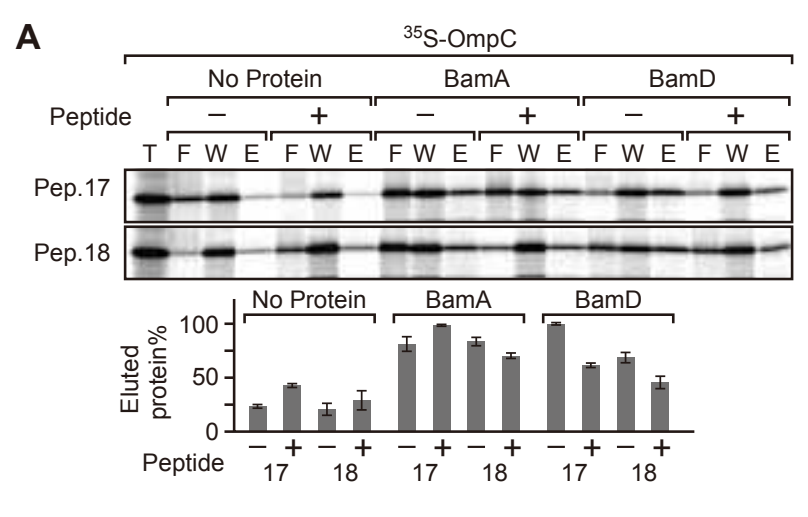

C
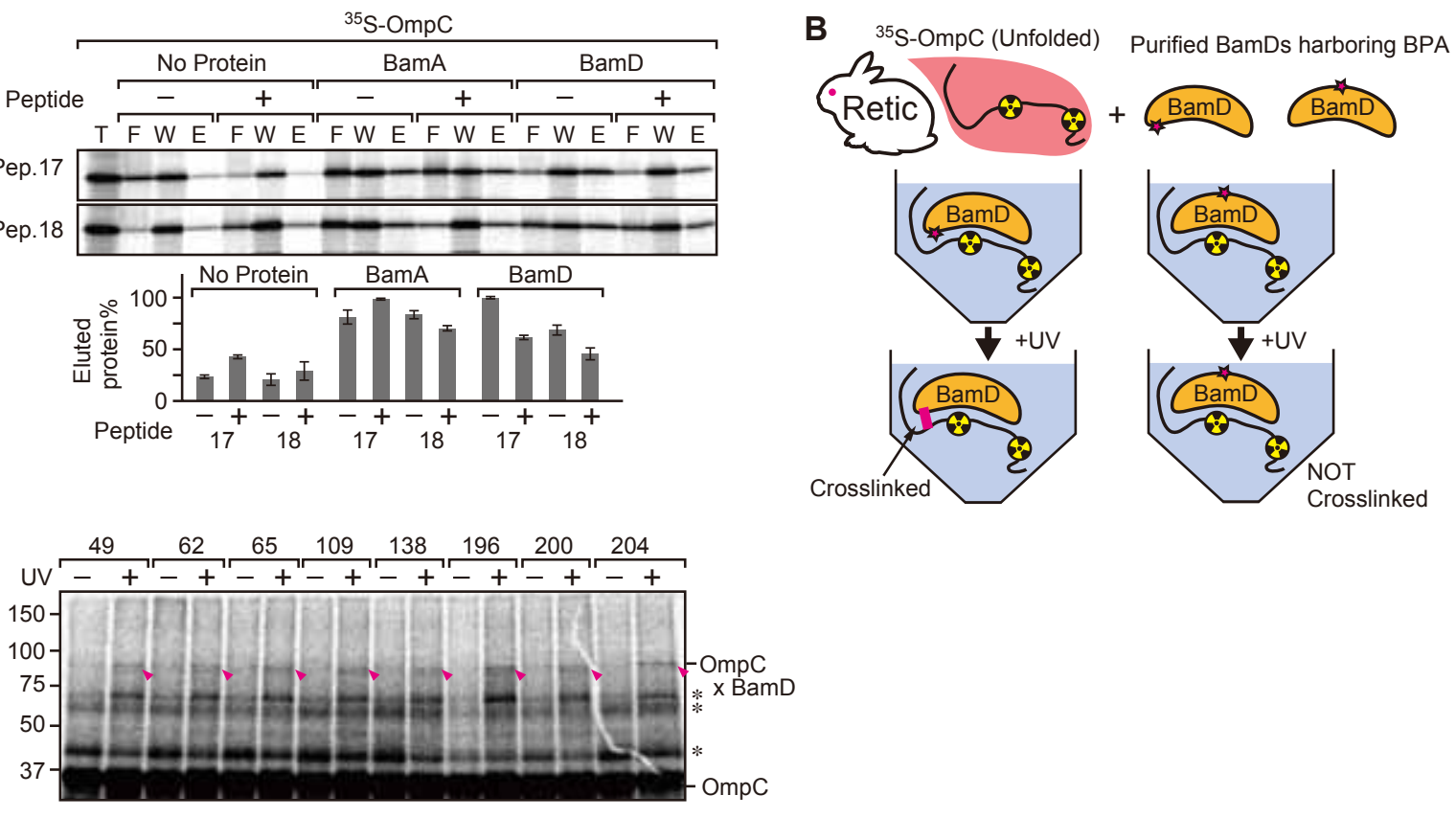

D
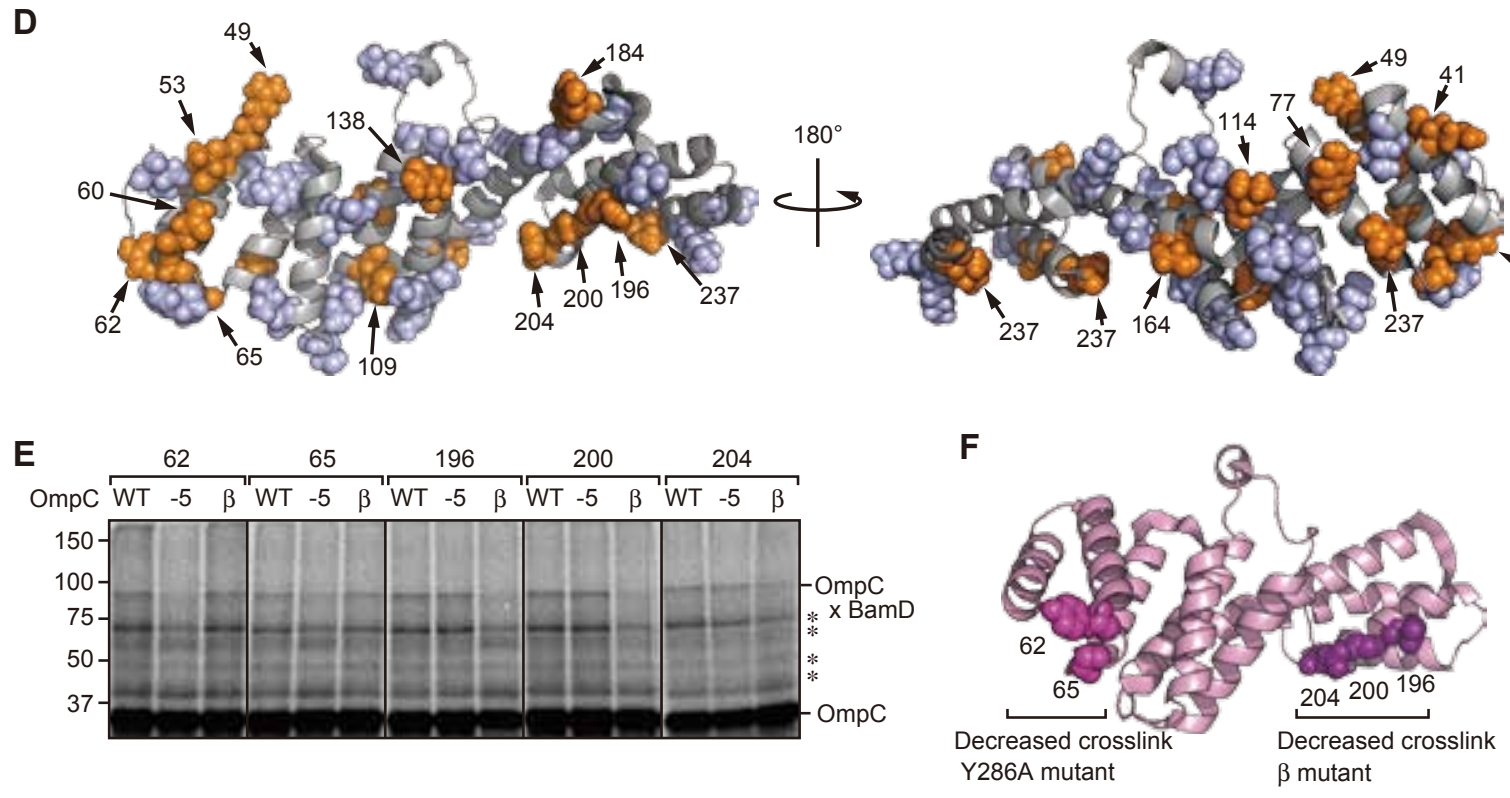

Germany et al. Fig. 4 
A

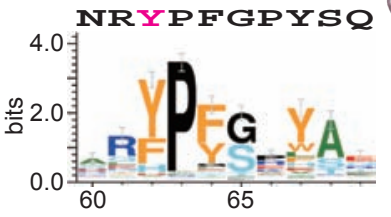

C

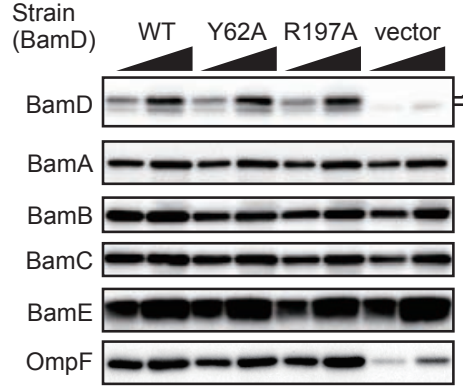

E

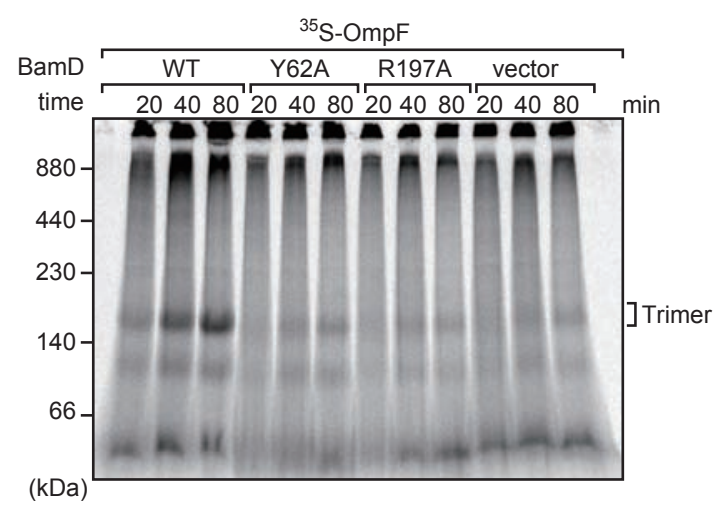

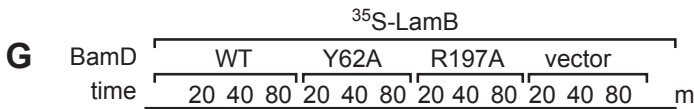

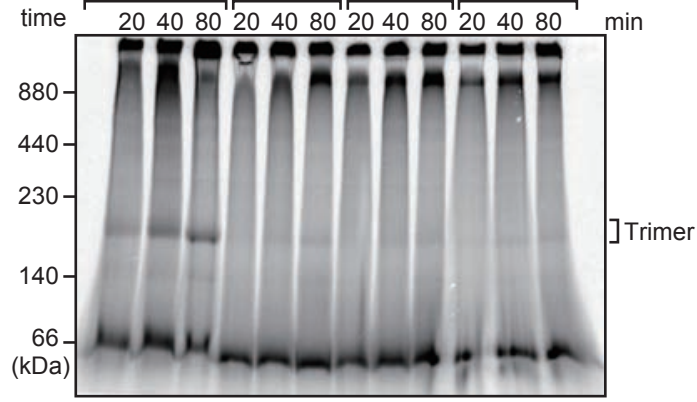

B

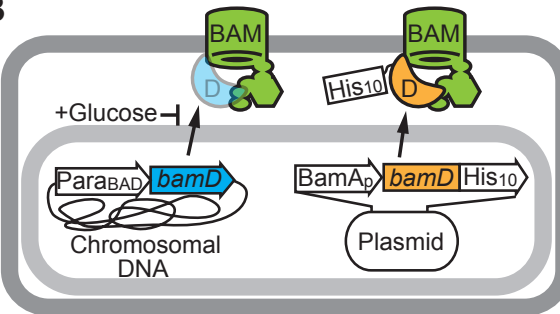

D

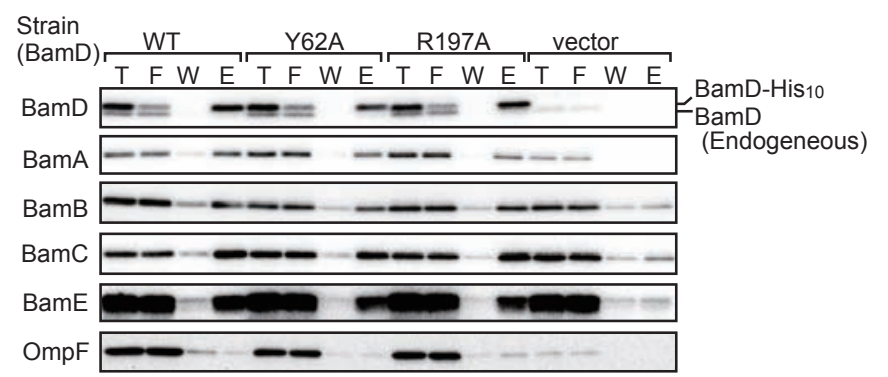

$\mathbf{F}$

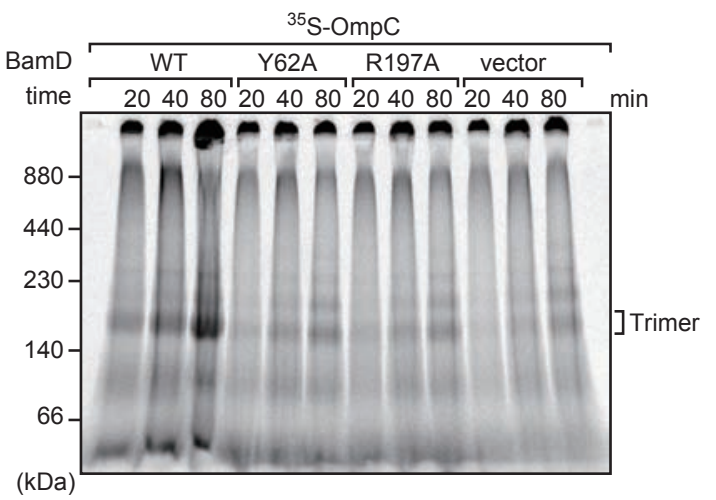

Germany et al. Fig. 5 
bioRxiv preprint doi: https://doi.org/10.1101/2021.10.29.466387; this version posted October 29, 2021. The copyright holder for this preprint (which was not certified by peer review) is the author/funder. All rights reserved. No reuse allowed without permission.

A

${ }^{35} \mathrm{~S}-\mathrm{OmpC}(\mathrm{Y} 286 \mathrm{~A})$

Antibody - +

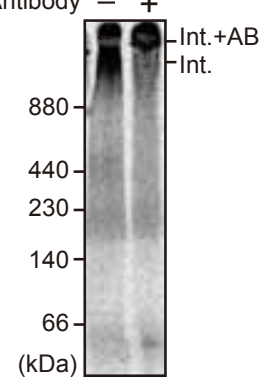

B

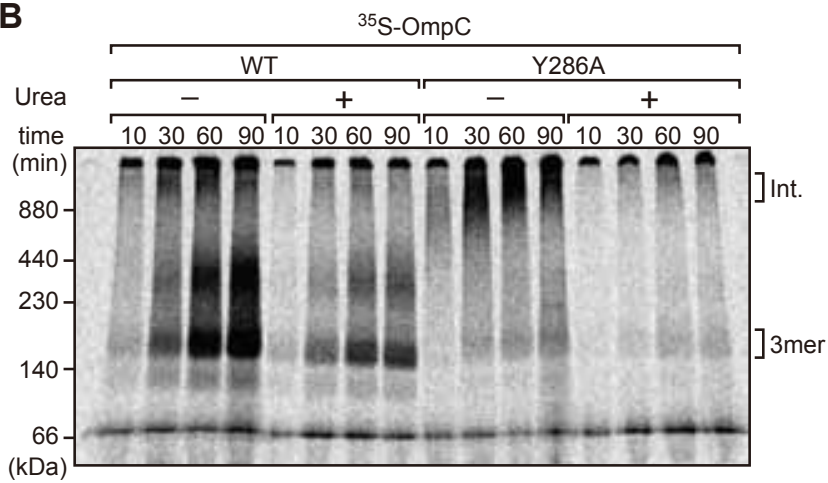

C

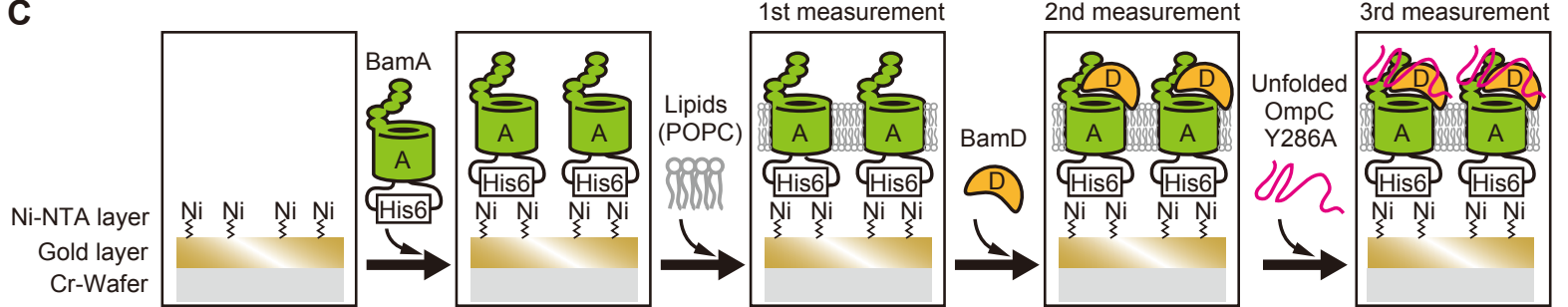

D

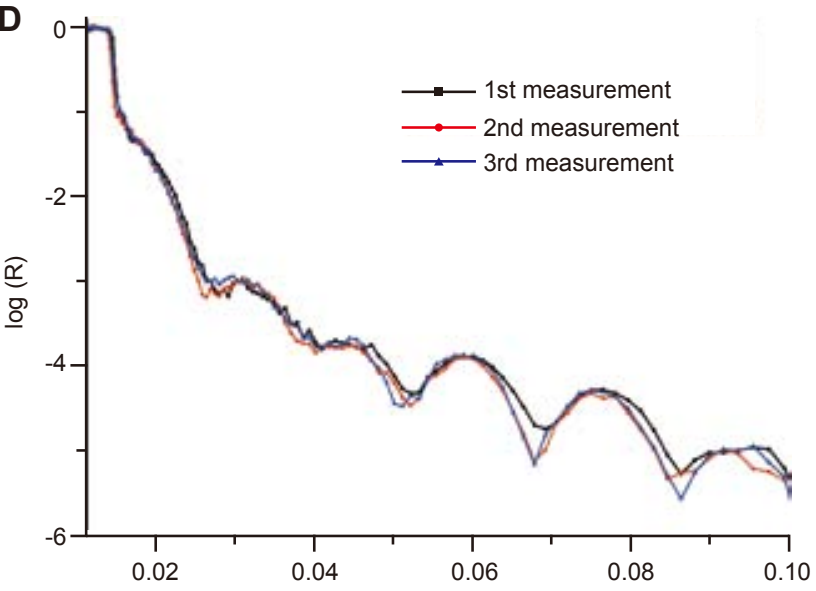

$\mathbf{F}$

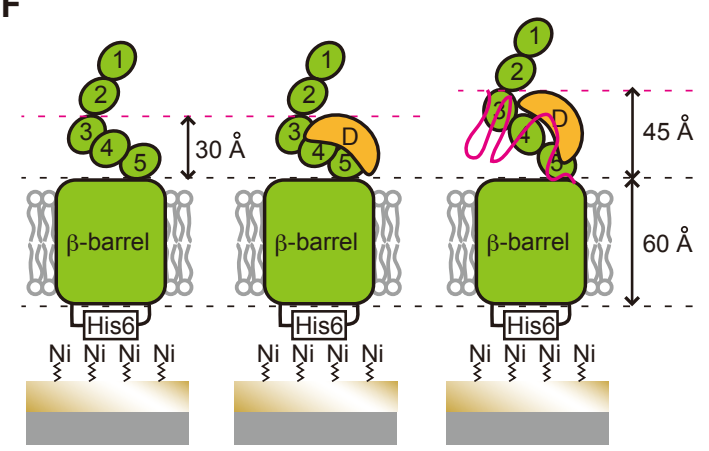

E

\begin{tabular}{l|lll|lll}
\multicolumn{1}{c}{ 1st measurement } & \multicolumn{3}{c}{ 2nd measurement } & \multicolumn{3}{c}{ Rrd measurement } \\
\hline Layers & \multicolumn{3}{|c}{ Thickness $(\AA)$} & \multicolumn{3}{c}{ Roughness $(\AA)$} \\
\hline His 8 & $6.0 \pm 0.5$ & $7.1 \pm 0.8$ & $9.4 \pm 0.5$ & $4.0 \pm 0.1$ & $4.0 \pm 0.1$ & $4.0 \pm 0.1$ \\
$\beta$-Barrel & $56.9 \pm 1.5$ & $58.7 \pm 0.4$ & $56.8 \pm 1.5$ & $10.1 \pm 0.1$ & $12.6 \pm 1.8$ & $6.0 \pm 0.1$ \\
P3-5 & $29.5 \pm 0.8$ & $29.7 \pm 0.8$ & $45.5 \pm 1.6$ & $7.0 \pm 0.1$ & $6.1 \pm 0.1$ & $12.1 \pm 0.1$ \\
P1-2 & $31.2 \pm 0.8$ & $30.3 \pm 0.4$ & $28.5 \pm 0.9$ & $13.8 \pm 0.1$ & $13.7 \pm 0.1$ & $5.0 \pm 0.1$ \\
\hline
\end{tabular}

\begin{tabular}{|c|c|c|c|c|c|c|c|c|c|c|c|c|}
\hline & & & & & & Volume & e fraction & (\%) & & & & \\
\hline & & BamA & & & POPC & i & Ban & & OmpC & & ution & \\
\hline $\mathrm{His}_{8}$ & --- & --- & --- & --- & --- & --- & --- & --- & --- & $25.3 \pm 7.4$ & $25.8 \pm 7.0$ & $22.7 \pm 1.7$ \\
\hline B-Barrel & $17.1 \pm 0.6$ & $18.4 \pm 1.1$ & $18.3 \pm 1.2$ & $35.1 \pm 1.6$ & $35.4 \pm 5.5$ & $35.9 \pm 1.5$ & --- & --- & --- & $47.6 \pm 2.9$ & $46.2 \pm 6.7$ & $45.8 \pm 2.7$ \\
\hline P3-5 & $10.7 \pm 0.9$ & $11.0 \pm 0.8$ & $11.7 \pm 2.9$ & $3.4 \pm 1.7$ & $3.3 \pm 0.5$ & $3.3 \pm 0.9$ & $8.8 \pm 1.1$ & $9.0 \pm 3.3$ & $8.0 \pm 1.2$ & $85.9 \pm 2.6$ & $76.9 \pm 2.0$ & $76.0 \pm 7.3$ \\
\hline $1-2$ & $11.2 \pm 0.8$ & $11.2 \pm 2.9$ & $10.7 \pm 0.9$ & --- & --- & --- & --- & --- & --- & $90.0 \pm 2.5$ & $88.8 \pm 2.9$ & $89.3 \pm 0.9$ \\
\hline
\end{tabular}


bioRxiv preprint doi: https://doi.org/10.1101/2021.10.29.466387; this version posted October 29, 2021. The copyright holder for this preprint (which was not certified by peer review) is the author/funder. All rights reserved. No reuse allowed without permission.

A

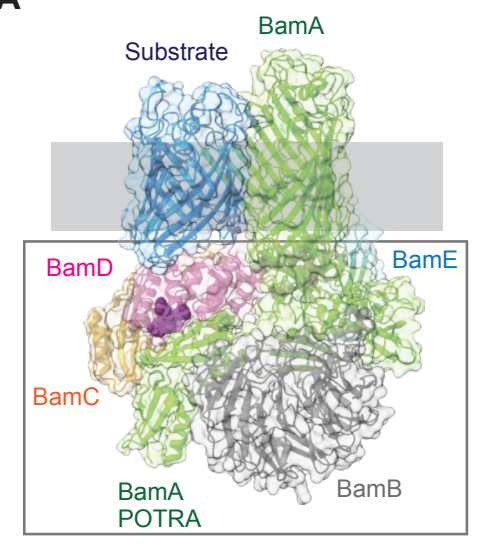

C

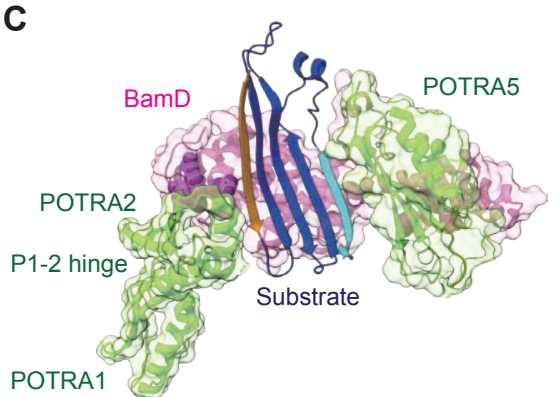

B

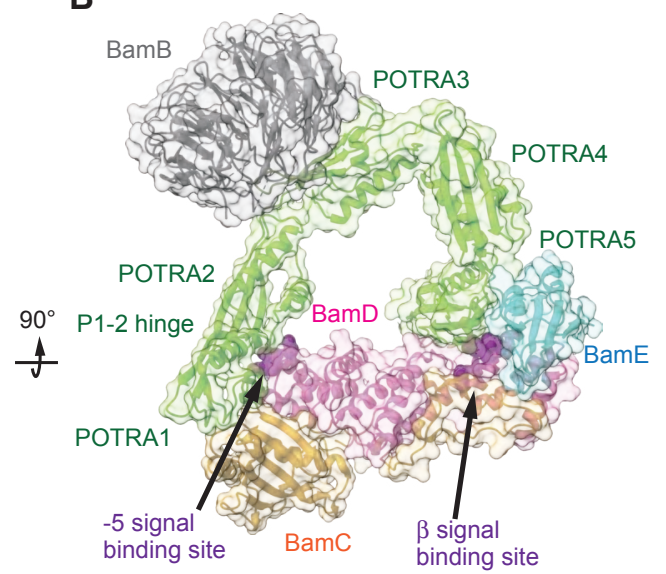

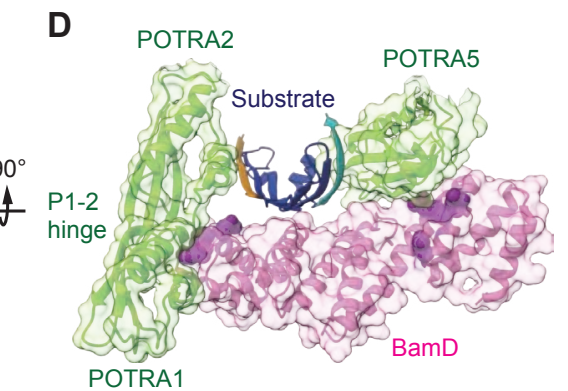

